\title{
On the numerical modeling of frictional wear phenomena
}

\author{
C. Agelet de Saracibar*, M. Chiumenti \\ ETS Ingenieros de Caminos, Canales y Puertos, International Center for Numerical Methods in Engineering, Edificio Cl, \\ Campus Norte, UPC, Gran Capitán s/n, 08034 Barcelona, Spain
}

Received 28 March 1998

\begin{abstract}
The evolution of the contact surfaces wear may become particularly important in the definition of the frictional behavior, in particular for frictional contact problems involving large slips, typically in sheet metal forming and bulk forming operations. Despite this fact, most of the current applications reported in the literature are restricted to a standard Coulomb law, using a constant friction coefficient. Such simple models may represent only a limited range of tribological situations and it appears to be necessary to develop a class of models which incorporate the state conditions and their evolution at the contact surfaces, taking into account the influence of complex phenomena such as wear, lubrication and chemical reactions, among others, see Oden and Martins [1].

In this paper a simple numerical model for the simulation of frictional wear behavior, within a fully nonlinear kinematic setting, including large slip and finite deformation, is presented. The model relies on the introduction of an internal variable related to the state conditions at the contact surface. Here, two possible definitions of this internal variable have been considered. The fully nonlinear frictional contact formulation, entirely derived first on a continuum setting by Laursen and Simo [2-6], has been extended here to accommodate the characterization of the wear frictional behavior.

Within the computational aspects, two families of robust time stepping algorithms, arising from an operator split of the constrained frictional evolution equations, are discussed.

Finally, following current approaches, see Lassen [9], Lassen and Bay [10], Owen et al. [11], de Souza et al. [12], Stromberg et al. [13] and Stromberg [14], a long-term tools wear prediction is given by introducing an a priori wear estimate derived from Archard's law, Archard [15].

The numerical model has been implemented into an enhanced version of the computational finite element program FEAP. Numerical examples show the suitability of the proposed model to capture the essential features of the frictional behavior at the contact interfaces and to provide a prediction of tool wear in forming operations. (c) 1999 Elsevier Science S.A. All rights reserved.
\end{abstract}

\section{Introduction. Motivation and goals}

Numerical analysis of frictional contact problems has been one of the research topics of main interest in recent years. Frictional contact problems arise in many application fields such as metal forming processes, crashworthiness and projectile impact, among others. In spite of important progresses achieved in the computational mechanics, the large scale numerical simulation of these topics continues, nowadays, to be a very complex task due mainly to the highly nonlinear nature of the problem, usually involving nonlinear kinematics, large deformations, large inelastic strains, nonlinear boundary conditions, frictional contact interaction, wear phenomena, large slips and in many cases coupled thermomechanical effects. During the last decade, growing interest on these and related topics, has been shown by many industrial companies, such as automotive and aeronautical, motivated by the need to get high quality final products and to reduce manufacturing costs.

Frictional behavior at the contact surfaces, between two bodies sliding relative to one another, highly depends on the nature and topography of the surfaces in contact, such as the hardness and the geometry of the microasperities, surface coating, etc. as well as on the environmental factors, such as the lubrication, which characterize the state conditions of the contact surfaces. These state conditions at the contact surfaces may be

\footnotetext{
* Corresponding author.
} 
constantly changing as a consequence of complex phenomena taking place during continuous sliding of the bodies, such as the wear due to the evolution of the surfaces topography, i.e. deformation of the microasperities, worn of surface coating, etc. internal straining, chemical reactions, etc. (see [1]).

The importance of the consideration of these wear phenomena in the characterization of the frictional behavior, clearly appears in two types of zinc coated sheet metals, the Galvannealed (GA) and Electrogalvanised (EG) steel sheets, typically employed in the manufacture of automotive body parts. In a GA steel sheet, the presence of a hard surface coating, difficult to remove, leads to a progressive softening of the frictional behavior, while in contrast an EG steel sheet experiences an initial softening, due to flattening of microasperities, followed by a substantial increase of the friction coefficient, due to the removal of its relatively soft zinc coat (see [12]).

Despite these facts, most of the current applications reported in the literature are restricted to a standard Coulomb law, using a constant friction coefficient. Such simple models may represent only a limited range of tribological situations and it appears to be necessary to develop a class of models which incorporate the state conditions and their evolution at the contact surfaces, taking into account the influence of complex phenomena such as wear, lubrication and chemical reactions, among others (see [1]).

In this paper a simple numerical model for the simulation of frictional wear behavior, within a fully nonlinear kinematic setting, including large slip and finite deformation, is presented. The model relies on the introduction of an internal variable related to the state conditions at the contact surface. Here, two possible definitions of this internal variable have been considered, identifying the internal variable as the (accumulated) frictional dissipation or as the slip distance. Both definitions have been incorporated, in a unified manner, into the model by replacing the constant friction coefficient of the classical Coulomb law by a (nonlinear) function of the internal variable. The fully nonlinear frictional contact formulation, entirely derived first on a continuum setting by Laursen and Simo [2-6], has been extended here, in Section 2, to accommodate the characterization of the wear frictional behavior.

Within the computational aspects, two families of robust time stepping algorithms, arising from an operator
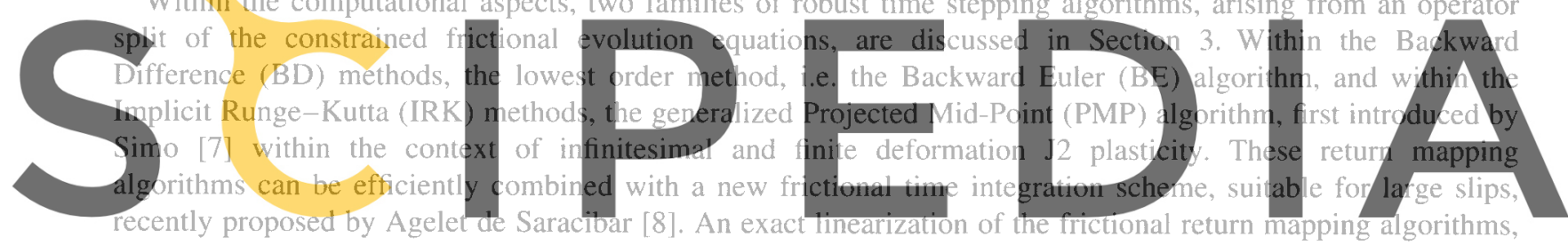

written in a unified form suitable for the BE and the PMP algorithms, has been performed and the consistent

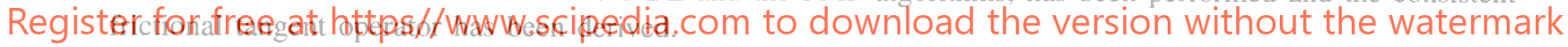

On the other hand, the phenomena related to wear on the tools have an important impact on the economy of industrial metal forming processes. Statistical results show that wear is the dominating die failure mechanism for both bulk and sheet forming operations and has therefore an important influence on the production costs of formed products. Methodologies currently used are based mainly on designers intuition and experience, which are not the most adequate when considering the complexity of the problem. Experts claim that a more quantitative approach to die design would improve service life considerably, leading to an important reduction of manufacturing costs for forging and stamping production. To enhance available decision support systems used in industrial design and optimization practice, taking into account wear phenomena, is one of the goals to be achieved in the up-coming years.

Section 4 deals with a summary of tools wear mechanisms, in which adhesive and abrasive wear are identified as the two most important wear mechanisms in hot forging and sheet metal forming processes. Here, following current approaches, see Lassen [9], Lassen and Bay [10], Owen et al. [11] and de Souza et al. [12], Stromberg et al. [13] and Stromberg [14], a (long-term) tools wear prediction is given by introducing an a priori (semi-empirical) instantaneous tool wear rate estimate derived from Archard's law [15]. Time integration of the wear rate estimate leads to an estimate of the accumulated tool wear over a larger number of forming cycles (see e.g. [9] and [11]).

The numerical model has been implemented into an enhanced version of the computational finite element program FEAP developed by R.L. Taylor and J.C. Simo and described [16]. Numerical examples, given in Section 5, show the suitability of the proposed model to capture the essential features of the frictional behavior at the contact interfaces and to provide a prediction of tools wear in forming operations. Finally, some concluding remarks are drawn. 


\section{Formulation of the frictional wear contact problem}

In this section we summarize the continuum formulation of the frictional contact problem first proposed by Laursen and Simo [2-6], here extended to accommodate wear phenomena through the introduction of an internal state variable, to be defined as the frictional dissipation or the slip amount, into the Coulomb frictional law.

\subsection{Frictional contact constrains}

\subsubsection{Notation}

Let $2 \leqslant n_{\mathrm{dim}} \leqslant 3$ be the space dimension and $I:=[0, T] \subset \mathbb{R}_{+}$the time interval of interest. Let the open sets $\Omega^{(1)} \subset \mathbb{R}^{n_{\mathrm{dim}}}$ and $\Omega^{(2)} \subset \mathbb{R}^{n \mathrm{dim}}$ with smooth boundaries $\partial \Omega^{(1)}$ and $\partial \Omega^{(2)}$ and closures $\bar{\Omega}^{(1)}:=\Omega^{(1)} \cup \Omega^{(1)}$ and $\bar{\Omega}^{(2)}:=\Omega^{(2)} \cup \partial \Omega^{(2)}$, be the reference placement of two continuum bodies $\mathscr{B}^{(1)}$ and $\mathscr{B}^{(2)}$, with material particles labeled $X \in \bar{\Omega}^{(1)}$ and $Y \in \bar{\Omega}^{(2)}$, respectively.

Denote by $\varphi^{(i)}: \bar{\Omega}^{(i)} \times I \rightarrow \mathbb{R}^{n}$ dim the orientation preserving deformation map of the body $\mathscr{B}^{(i)}$, with material velocities $V^{(i)}:=\partial_{t} \varphi^{(i)}$ and deformation gradients $F^{(i)}:=D \varphi^{(i)}$. For each time $t \in I$, the mapping $t \in$ $I \mapsto \varphi_{i}^{(i)}:=\varphi^{(i)}(\cdot, t)$ represents a one-parameter family of configurations indexed by time $t$, which maps the reference placement of body $\mathscr{B}^{(i)}$ onto its current placement $\mathscr{S}_{i}^{(i)}:=\varphi_{i}^{(i)}\left(\mathscr{B}^{(i)}\right) \subset \mathbb{R}^{\prime \prime}$ dim.

We will denote as the contact surface $\Gamma^{(i)} \subset \partial \Omega^{(i)}$ the part of the boundary of the body $\mathscr{B}^{(i)}$ such that all material points where contact will occur at any time $t \in I$ are included. The current placement of the contact surface $\Gamma^{(i)}$ is given by $\gamma^{(i)}:=\varphi_{i}^{(i)}\left(\Gamma^{(i)}\right)$.

Attention will be focussed to material points on these surfaces denoted as $X \in \Gamma^{(1)}$ and $Y \in \Gamma^{(2)}$. Current placement of these particles is given by $x=\varphi_{i}^{(1)}(\boldsymbol{X}) \in \gamma^{(1)}$ and $y=\varphi_{i}^{(2)}(\boldsymbol{Y}) \in \gamma^{(2)}$. See Fig. 1 for an illustration
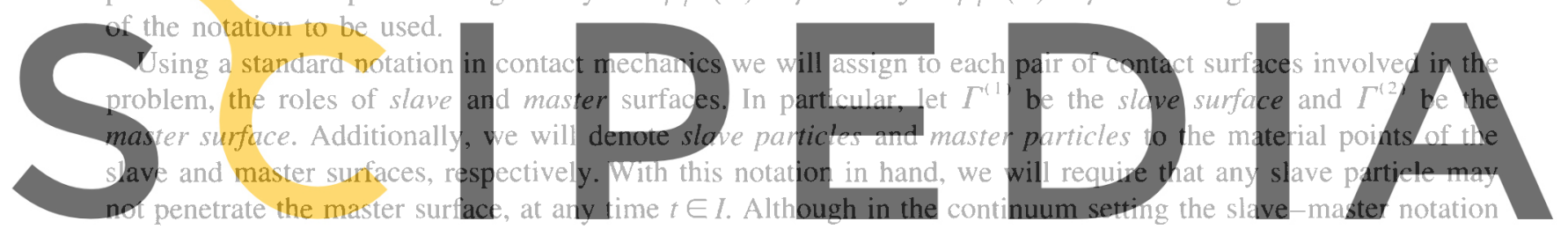

plays no role, in the discrete setting this choice becomes important.

Register for free at https//www.scipedia.com to download the version without the watermark

2.1.2. Parametrization of the contact surfaces

Let $\mathscr{A}^{(i)} \subset \mathbb{R}^{n d \mathrm{dim}-1}$ be a parent domain for the contact surface of body $\mathscr{B}^{(i)}$. A parametrization of the contact surface for each body $\mathscr{B}^{(i)}$ is introduced by a family of (orientation preserving) one-parameter mappings indexed by time, $\boldsymbol{\psi}_{i}^{(i)}: \mathscr{A}^{(i)} \subset \mathbb{R}^{n_{\mathrm{dim}}{ }^{-1}} \rightarrow \mathbb{R}^{n \text { dim }}$ such that $\Gamma^{(i)}:=\boldsymbol{\psi}_{0}^{(i)}\left(\mathscr{A}^{(i)}\right)$ and $\gamma^{(i)}:=\boldsymbol{\psi}_{t}^{(i)}\left(\mathscr{A}^{(i)}\right)$. Using the mapping composition rule, it also follows that $\boldsymbol{\psi}_{t}^{(i)}=\boldsymbol{\varphi}_{1}^{(i)} \circ \boldsymbol{\psi}_{0}^{(i)}$. It will be assumed in what follows that these parametrizations have the required smoothness conditions.

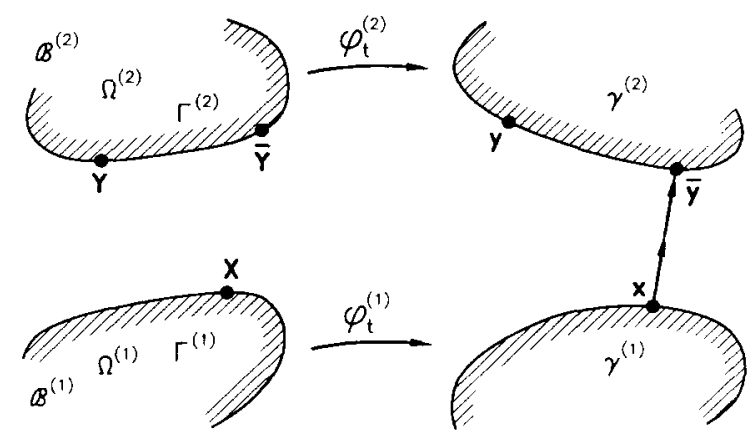

Fig. 1. Schematic description of two interacting bodies at reference and current placements. Reference and current placement of contact surfaces. 
Within the slave-master surface role, focus will be placed on the parametrization of the master surface. Using the parametrization of the contact surfaces introduced above we consider a point $\xi:=\left(\xi^{1}, \xi^{2}\right) \in \mathscr{A}^{(2)}$ of the parent domain, such that

$$
\begin{aligned}
& \boldsymbol{Y}:=\boldsymbol{\psi}_{0}^{(2)}(\xi), \quad y:=\boldsymbol{\psi}_{t}^{(2)}(\xi) \\
& E_{c \gamma}(\xi):=\boldsymbol{\psi}_{0, \alpha}^{(2)}(\xi), \quad \boldsymbol{e}_{\alpha}(\xi):=\boldsymbol{\psi}_{t, \alpha}^{(2)}(\xi)
\end{aligned}
$$

where $\boldsymbol{Y}$ and $\boldsymbol{y}$ are, respectively, the reference and current placement of a master particle and $\boldsymbol{E}_{\alpha}(\boldsymbol{\xi})$ and $\boldsymbol{e}_{\alpha}(\boldsymbol{\xi})$, $\alpha=1,2$ are the convected surface basis attached to the master particle $Y \in \Gamma^{(2)}$, on the reference and current configuration, respectively. Here, $(\cdot)_{\alpha}$ denotes partial derivative with respect to $\xi^{\alpha}$. Fig. 2 shows the parametrization map of reference and current placement of a contact surface.

\subsubsection{Contact normal constraints}

Let $\bar{y}(\boldsymbol{X}, t) \in \gamma^{(2)}$ be the closest-point projection of the current position of the slave particle $\boldsymbol{X}$ onto the current placement of the master surface $\Gamma^{(2)}$, defined as

$$
\overline{\boldsymbol{Y}}(\boldsymbol{X}, t):=\arg \min _{\boldsymbol{Y} \in \Gamma^{(2)}}\left\{\left\|\varphi_{i}^{(1)}(\boldsymbol{X})-\varphi_{i}^{(2)}(\boldsymbol{Y})\right\|\right\}, \quad \bar{y}(\boldsymbol{X}, t):=\varphi_{i}^{(2)}(\overline{\boldsymbol{Y}})
$$

and let $g_{N}(\boldsymbol{X}, t)$ be the gap function defined for any slave particle $X \in \Gamma^{(1)}$ and for any time $t \in I$ as

$$
g_{N}(X, t):=-\left[\varphi_{1}^{(1)}(\boldsymbol{X})-\varphi_{1}^{(2)}(\overline{\boldsymbol{Y}}(\boldsymbol{X}, t))\right] \cdot \nu
$$

where $\nu:=\gamma^{(2)} \rightarrow S^{2}$ is the unit outward normal field to the current placement of the master surface particularized at the closest-point projection $\bar{y}(X, t) \in \gamma^{(2)}$.

Let $\mathbb{P}^{(1)}(\mathbb{X}, t)$ be the first Piola-Kirchhoff stress tensor and $N^{(1)}(\mathbb{X})$ the unit outward normal to the slave
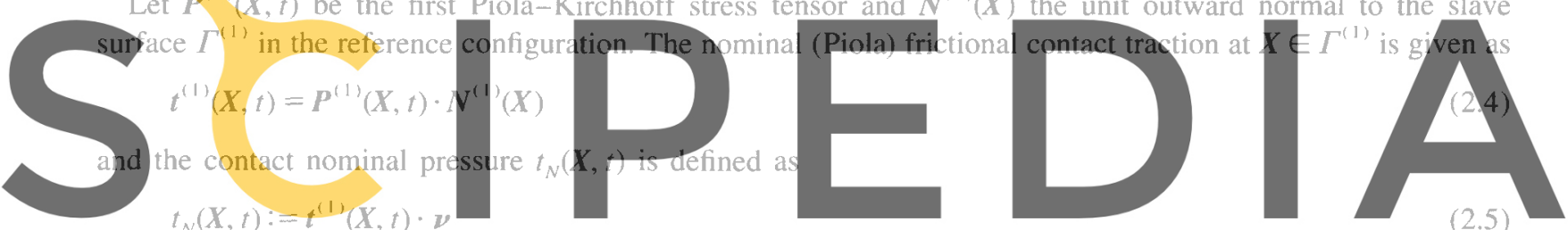

$t_{N}(\mathbb{X}, t):=t^{(1)}(\boldsymbol{X}, t) \cdot \boldsymbol{\nu}$

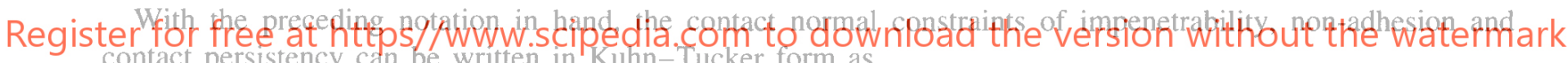 \\ contact persistency can be written in Kuhn-Tucker form as}

$$
\begin{aligned}
& t_{N} \geqslant 0, \quad g_{N} \leqslant 0, \quad t_{N} g_{N}=0 \\
& t_{N} \dot{g}_{N}=0 \quad \text { iff } t_{N}>0
\end{aligned}
$$

\subsubsection{Convected basis, metric and curvature tensors at the closest-point projection}

Associated to the closest-point projection given by (2.2), for some point $\bar{\xi}:=\left(\bar{\xi}^{1}, \bar{\xi}^{2}\right) \in \mathscr{A}^{(2)}$ of the parent domain we will have

$$
\overline{\boldsymbol{Y}}(X, t):=\boldsymbol{\psi}_{0}^{(2)}(\bar{\xi}(X, t)), \quad \overline{\boldsymbol{y}}(\boldsymbol{X}, t):=\boldsymbol{\psi}_{t}^{(2)}(\bar{\xi}(X, t))
$$

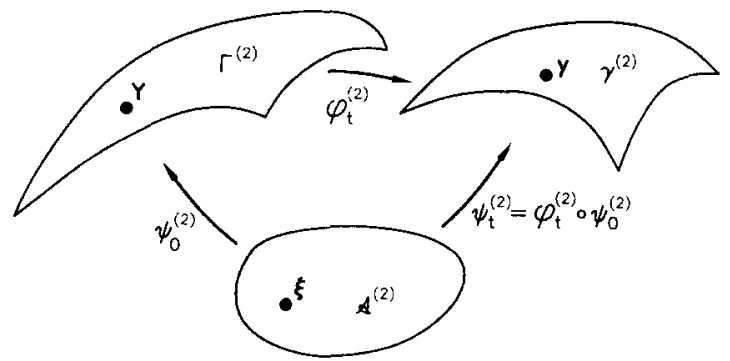

Fig. 2. Contact surfaces parametrization. Parametrization map of reference and current placement of a contact surface 
Attached to the master particle $\bar{Y}(X, t) \in \Gamma^{(2)}$ we define the convected surface basis on the reference and current configurations, respectively, as

$$
\tau_{\alpha}^{\mathrm{ref}}(\boldsymbol{X}, t):=\boldsymbol{E}_{\alpha}(\bar{\xi}(X, t)), \quad \boldsymbol{\tau}_{\alpha}(\boldsymbol{X}, t):=\boldsymbol{e}_{\alpha}(\bar{\xi}(X, t))
$$

Additionally, the unit outward normals $\nu^{\text {ref }} \in S^{2}$ and $\nu \in S^{2}$ at the master particle $\overline{\boldsymbol{Y}}(\boldsymbol{X}, t)$ on the reference and current configurations, respectively, can be defined as

$$
\nu^{\mathrm{ref}}:=\frac{\tau_{1}^{\mathrm{ref}} \times \tau_{2}^{\mathrm{ref}}}{\left\|\tau_{1}^{\mathrm{ref}} \times \tau_{2}^{\mathrm{ref}}\right\|}, \quad \boldsymbol{\nu}:=\frac{\tau_{1} \times \tau_{2}}{\left\|\tau_{1} \times \tau_{2}\right\|}
$$

The vectors $\tau_{\alpha}^{\text {ref }} \in T_{\nu \text { ret }} S^{2}$ and $\tau_{\alpha} \in T_{\nu} S^{2}, \alpha=1,2$ span the tangent spaces $T_{\nu \text { ret }} S^{2}$ and $T_{\nu} S^{2}$ to the $S^{2}$ unit sphere at $\nu^{\text {ref }}$ and $\nu$, respectively. Here, the tangent space to the $S^{2}$ unit sphere at $\nu \in S^{2}$ is defined as

$$
T_{\nu} S^{2}:=\left\{\delta \boldsymbol{\nu} \in \mathbb{R}^{n_{\mathrm{dim}}}: \delta \boldsymbol{\nu} \cdot \boldsymbol{\nu}=0\right\}
$$

The convected surface basis vectors $\boldsymbol{\tau}_{\alpha}^{\mathrm{ref}}$ and $\boldsymbol{\tau}_{\alpha}, \alpha=1,2$, augmented with the unit outward normals $\boldsymbol{\nu}^{\text {ref }}$ and $\boldsymbol{\nu}$, provides local spatial frames at the master particle $\overline{\boldsymbol{Y}}(\boldsymbol{X}, t)$ on the reference and current configurations, respectively.

The convected surface basis vectors $\tau_{\alpha}^{\mathrm{ref}}$ and $\tau_{\alpha}, \alpha=1,2$, induces a surface metric or first fundamental form on the reference and current configurations, defined, respectively, as

$$
M_{\alpha \beta}:=\tau_{\alpha}^{\mathrm{ref}} \cdot \tau_{\beta}^{\mathrm{ref}}, \quad m_{\alpha \beta}:=\tau_{\alpha} \cdot \tau_{\beta}
$$

Inverse surface metrics $M^{\alpha \beta}$ and $m^{\alpha \beta}$ are defined in the usual manner. Additionally, dual surface basis on the reference and current configurations are straightforward defined, respectively, as

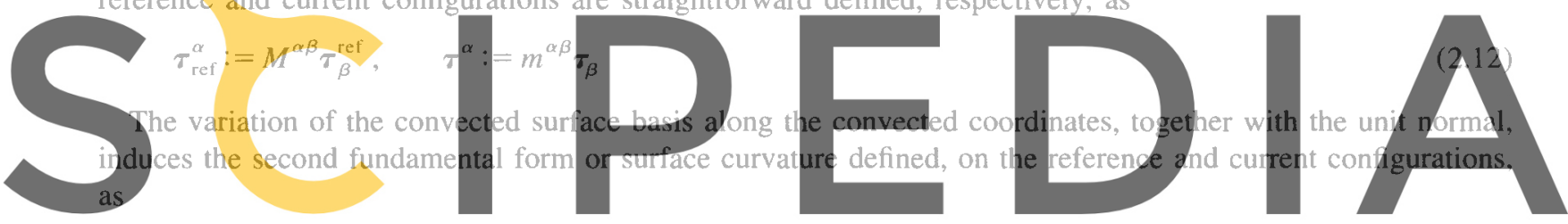

\section{$\kappa_{\alpha \beta \beta}^{\mathrm{ref}}:=\boldsymbol{E}_{\alpha, \beta}(\bar{\xi}) \cdot \nu^{\mathrm{ref}}, \quad \kappa_{\alpha \beta}:=\boldsymbol{e}_{\alpha, \beta}(\bar{\xi}) \cdot \boldsymbol{\nu}$}

Register for free at https//Www.scipedia.com to download the version without the watermark

\subsubsection{Constrained frictional evolution problem}

The relative slip velocities in the convected and current configuration are defined as

$$
\boldsymbol{v}_{T}^{\mathrm{ref}}(\boldsymbol{X}, t):=\dot{\overline{\boldsymbol{Y}}}(\boldsymbol{X}, t), \quad \boldsymbol{v}_{T}(\boldsymbol{X}, t):=\boldsymbol{F}_{t}^{(2)}(\overline{\boldsymbol{\xi}}(\boldsymbol{X}, t)) \cdot \boldsymbol{v}_{T}^{\mathrm{ref}}(\boldsymbol{X}, t)
$$

and, applying the chain rule,

$$
\boldsymbol{v}_{T}^{\mathrm{ref}}(\boldsymbol{X}, t):=\underline{\xi}^{\alpha} \tau_{\alpha}^{\mathrm{ref}}, \quad \boldsymbol{v}_{T}(\boldsymbol{X}, t):=\underline{\xi}^{\alpha} \tau_{\alpha}
$$

The one-form associated to these objects are defined as

$$
\boldsymbol{v}^{\mathrm{refl}}(X, t):=\underline{\xi}^{\alpha} M_{\alpha \beta} \tau_{\text {ref }}^{\beta}, \quad v_{T}^{b}(X, t):=\underline{\xi}^{\alpha \alpha} M_{\alpha \beta} \tau^{\beta}
$$

We define the nominal frictional tangent traction $t_{T}(X, t)$ as (minus) the projection of the nominal frictional contact traction $t^{(1)}(X, t)$ onto the unit normal $\nu$, as

$$
\boldsymbol{t}_{T}(\boldsymbol{X}, t):=-\mathbb{P} t^{(1)}(X, t)=t_{T}^{\alpha}(X, t) \tau_{\alpha}
$$

Additionally, to take into account the short term wear behaviour, we introduce an internal variable, denoted by $\alpha(X, t)$, to be defined as the accumulated frictional dissipation, the slip amount or, eventually, a combination of both parameters.

With the preceding notation in hand the slip rule, internal evolution equation and frictional constraints can be written in Kuhn-Tucker form as 


$$
\begin{aligned}
& \boldsymbol{v}_{T}^{b}(\boldsymbol{X}, t)=\gamma \boldsymbol{p}_{T}^{b} \\
& \dot{\alpha}(\boldsymbol{X}, t)=\gamma\left[(1-w)+w\left\|\boldsymbol{t}_{T}^{b}\right\|\right] \\
& \Phi\left(\boldsymbol{t}_{T}^{b}, t_{N}, \alpha\right) \leqslant 0, \quad \gamma \geqslant 0, \quad \gamma \Phi\left(t_{T}^{b}, t_{N}, \alpha\right)=0 \\
& \gamma \dot{\Phi}\left(\boldsymbol{t}_{T}^{b}, t_{N}, \alpha\right)=0 \quad \text { if } \Phi\left(t_{T}^{b}, t_{N}, \alpha\right)=0
\end{aligned}
$$

where $\Phi\left(t_{T}^{b}, t_{N}, \alpha\right):=\left\|t_{T_{n+1}}^{b}\right\|-\mu\left(\alpha_{n+1}\right) t_{N_{n+1}}$ is the slip function defining the admissible nominal traction states, $\boldsymbol{p}_{T}^{b}$ is the gradient of the slip potential, usually defined as the slip function for constant pressure, $\alpha$ is an internal variable, defined as the frictional dissipation for $w=1$, the slip amount for $w=0$ or a combination of both parameters for an intermediate value of $w \in(0,1), \gamma$ is the slip rate and $(\cdot)^{b}$ states for the one-form associated to the corresponding vector object.

\subsection{Regularized frictional contact constraints}

As discussed in [17], for instance, solution of initial boundary value problems (IBVP) subject to constraints such as (2.6) and (2.16)-(2.17) amounts to finding a solution within a constrained solution space. Consideration of corresponding weak forms induces limitations on admissible variations in the tangent solution space, imposed by the physical constraints, leading to variational inequalities (see e.g. [17] or [18].)

Different methods have been used to bypass the need to find a solution within a constrained configuration solution space $[1-6,8]$. Here we will use the penalty method to remove the restrictions associated to the constrained solution space and enforce the constraints through the introduction of a constitutive-like equation for the frictional contact traction.

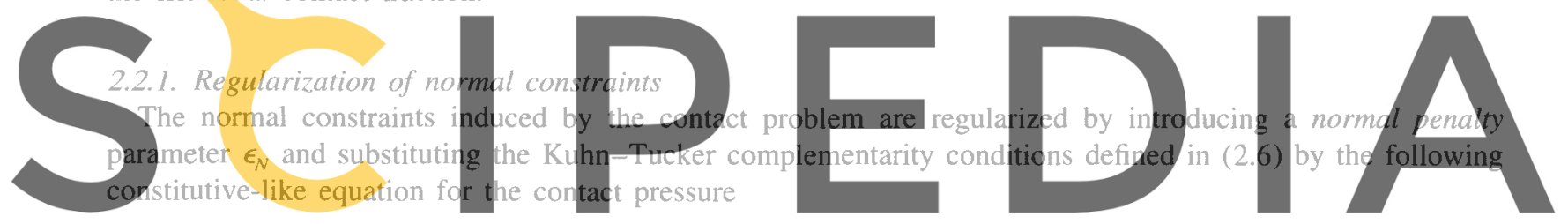

Register for free atthtspskwwyw.\$cipedia.com to download the version without the waterimark

where $\langle\cdot\rangle$ is the Macauley bracket, representing the positive part of its operand.

\subsubsection{Regularization of frictional constraints}

The regularization of the constrained frictional evolution problem defined by $(2.16)-(2.17)$ is performed introducing a tangential penalty parameter $\epsilon_{T}$ playing the role of constitutive parameter in the relative slip velocity evolution equation, leading to the following frictional constrained evolution problem

$$
\begin{aligned}
& \mathscr{L}_{v_{T}} \boldsymbol{t}_{T}^{b}=\epsilon_{T}\left[\boldsymbol{v}_{T}^{b}-\gamma \partial_{t_{T}^{b}} \Phi\left(t_{T}^{b}, t_{N}, \alpha\right)\right] \\
& \dot{\alpha}=\gamma\left[(1-w)+w\left\|\boldsymbol{t}_{T}^{b}\right\|\right] \\
& \Phi\left(\boldsymbol{t}_{T}^{b}, t_{N}, \alpha\right) \leqslant 0, \quad \gamma \geqslant 0, \quad \gamma \Phi\left(t_{T}^{b}, t_{N}, \alpha\right)=0 \\
& \gamma \dot{\Phi}\left(t_{T}^{b}, t_{N}, \alpha\right)=0
\end{aligned}
$$

where $\mathscr{L}_{v_{T}} \boldsymbol{t}_{T}^{b}:=\dot{t}_{T \alpha} \tau^{\alpha}$ is the Lie derivative of the frictional tangent traction along the flow induced by the relative slip velocity $\boldsymbol{v}_{T}$.

Within the context of the product formula algorithms, a frictional operator split of the constrained evolution problem can be introduced by means of a trial state, defined by freezing the irreversible (plastic) slip response, i.e. setting $\gamma=0$, as follows: 
Trial state

$$
\left\{\begin{array}{l}
\mathscr{L}_{v_{T}} t_{T}^{b}:=\epsilon_{T} v_{T}^{b} \\
\dot{\alpha}:=0 \\
\text { unconstrained }
\end{array}\right\} \quad\left\{\begin{array}{l}
\mathscr{L}_{v_{T}} t_{T}^{b}:=-\epsilon_{T} \gamma \partial_{t_{T}^{b}} \Phi\left(t_{T}^{b}, t_{N}, \alpha\right) \\
\dot{\alpha}:=\gamma\left[(1-w)+w\left\|t_{T}^{b}\right\|\right] \\
\Phi\left(t_{T}^{b}, t_{N}, \alpha\right) \leqslant 0, \quad \gamma \geqslant 0, \quad \gamma \Phi\left(t_{T}^{b}, t_{N}, \alpha\right)=0
\end{array}\right\}
$$

\subsection{Frictional contact contribution to the weak form of the momentum balance equation}

The frictional contact contribution to the weak form of the momentum balance equation can be conveniently expressed as [4]

$$
G_{c}:=\left\langle t_{N}, \delta g_{N}\right\rangle_{\Gamma^{(1)}}+\left\langle t_{T \alpha}, \delta \bar{\xi}^{\alpha}\right\rangle_{\Gamma^{(1)}}
$$

where $\delta[\cdot]$ is an admissible variation of its argument and $\left\langle\cdot \vartheta_{\Gamma^{(1)}}\right.$ denotes the $L_{2}\left(\Gamma^{(1)}\right)$-inner product on the boundary $\Gamma^{(1)}$. The expressions of the variations $\delta g_{N}$ and $\delta \xi^{\alpha}$ can be found in [4] and therefore will not be given here. The reader is referred to [2-6] for further details on local governing equations, boundary conditions, equilibrium equations at the contact interface and the weak form expressions for a multi-body frictional contact problem at finite deformations, as well as how to derive the compact form of the frictional contact contribution to the weak form of momentum balance equation given by $(2.21)$.
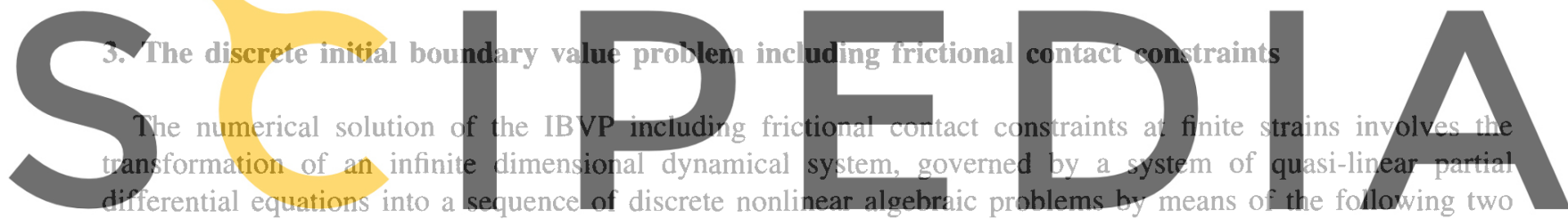

steps:

Register for free at https//www.scipedia.com to download the version without the watermark

Step 1. A finite element Galerkin projection in space of the dynamic weak form of the momentum equations leads to a nonlinear coupled system of ordinary differential equations (ODEs) which describe the time evolution of nodal degrees of freedom in the time interval of interest $I$.

Step 2. The coupled system of nonlinear ordinary differential equations describes the time evolution in the time interval $I$ of interest, of the nodal degrees of freedom and the internal variables associated with the finite element Galerkin projection. A time discretization of this problem involves a partition $I=\cup_{n=0}^{N}\left[t_{n}, t_{n+1}\right]$ of the time interval $I$. Within a typical time subinterval $\left[t_{n}, t_{n+1}\right]$, a time marching scheme for the advancement of the configuration and velocity fields together with a return mapping algorithm for the advancement of the internal variables results in a nonlinear algebraic problem which is solved iteratively.

\subsection{Spatial discretization: the Galerkin projection}

3.1.1. Galerkin projection of the frictional contact contribution to the weak form

The Galerkin projection of the frictional contact contribution to the weak form, given for the continuum case by $(2.21)$, can be written as

$$
G_{c}^{h}:=\left\langle t_{N}^{h}, \delta g_{N}^{h}\right\rangle_{\left.\Gamma^{(1)}\right)^{h}}+\left\langle t_{T \alpha}^{h}, \delta \bar{\xi}^{\alpha h}\right\rangle_{\Gamma^{(1)^{h}}}
$$

where $(\cdot)^{h}$ denotes the Galerkin projection of $(\cdot)$. 


\subsection{Temporal discretization. Frictional return mapping}

Consider the time interval of interest $I=[0, T]$ discretized into a series of non-overlapping subintervals $I:=\cup_{n=0}^{N}\left[t_{n}, t_{n+1}\right]$. The incremental solution to the IBVP is obtained applying a time stepping algorithm to integrate the evolution equations within a typical time step $\left[t_{n}, t_{n+1}\right]$, with given nodal and internal variables at time $t_{n}$, as initial conditions at the nodal and quadrature points of a typical element $\Omega_{a}^{(i)}$, respectively.

Following a standard convention, we shall denote by either $(\cdot)_{n}$ or $(\cdot)_{n+1}$ the algorithmic approximations at times $t_{n}$ and $t_{n+1}$ to the continuum (time dependent) variable $(\cdot)_{t}$.

\subsubsection{Frictional time-stepping algorithms}

Most of the usual time-stepping algorithms will require the evaluation of the weak form and internal variables at some time $t_{n+\vartheta}$, where $\vartheta \in(0,1]$. Here, attention will be restricted to the Backward-Euler algorithm, obtained for $\vartheta=1$. A class of time-stepping algorithms for dynamic plasticity, including Linear Multistep (LMS) methods and amongst them, the so-called BD methods, and IRK methods, are shown in [7,19]. Here, we will be focussed on two algorithms for the time integration of the constrained frictional evolution problem defined by (2.20): the lowest order BD method called BE method and the generalized PMP IRK method.

3.2.1.1. BE method. Consider the approximation of (2.16) and (2.17) by the lowest order BD method, the BE scheme, to obtain the algebraic equation

$t_{T_{\alpha, n+1}}=t_{T_{\alpha, n}}+\epsilon_{T}\left[M_{\alpha \beta}\left(\bar{\xi}_{n+1}^{\beta}-\bar{\xi}_{n}^{\beta}\right)-\gamma_{n+1} p_{T_{\alpha, n+1}}\right]$

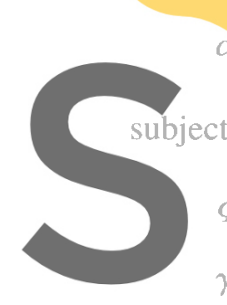

$\alpha_{n+1}=\alpha_{n}+\gamma_{n+1}\left[(1-w)+w \mid t_{T}^{b}\right.$
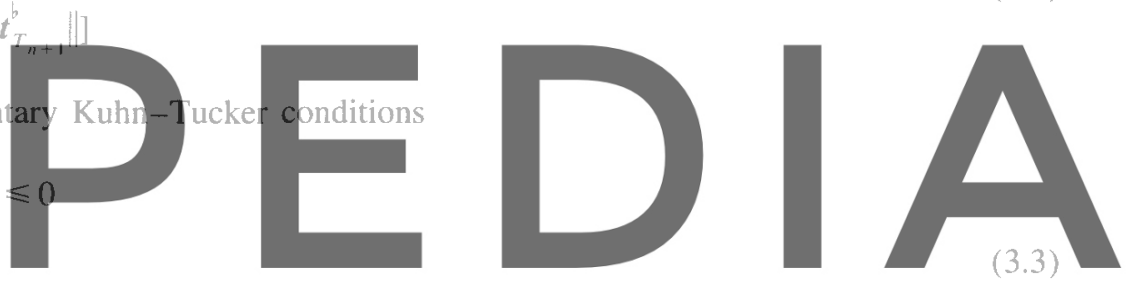

Register for free at https//www.scipedia.com to download the version without the watermark

The solution to the constrained incremental algebraic problem defined by (3.2) and (3.3) is obtained through the introduction of a trial state, obtained by freezing the irreversible-slip response, and subsequent return mapping algorithm to enforce the constraints.

Step 1: Trial state. The frictional trial state is obtained by freezing the irreversible-slip response, i.e. assuming $\gamma_{n+1}=0$ and that no constraints are present. Then, the trial state is defined as

$$
\begin{aligned}
& t_{T_{\alpha, n+1}^{\text {trial }}}:=t_{T_{\alpha, n}}+\epsilon_{T} M_{\alpha \beta}\left(\bar{\xi}_{n+1}^{\beta}-\bar{\xi}_{n}^{\beta}\right) \\
& \alpha_{n+1}^{\text {trial }}:=\alpha_{n} \\
& \Phi_{n+1}^{\text {trial }}:=\left\|t_{n+1}^{\text {trial }}\right\|-\mu\left(\alpha_{n+1}^{\text {trial }}\right) t_{N n+1}
\end{aligned}
$$

where $t_{N_{n+1}}=\epsilon_{N}\left\langle g_{N_{n+1}}\right\rangle$ is the normal contact pressure at $t_{n+1}$.

Step 2: Return mapping. The return mapping defines the final state as the solution of the discrete constrained incremental algebraic problem:

$$
\begin{aligned}
& t_{T_{\alpha, n+1}}=t_{T_{\alpha, n+1}^{\text {trial }}}-\epsilon_{T} \gamma_{n+1} p_{T_{\alpha, n+1}} \\
& \alpha_{n+1}=\alpha_{n+1}^{\text {trial }}+\gamma_{n+1}\left[(1-w)+w\left\|t_{T_{n+1}}^{b}\right\|\right]
\end{aligned}
$$




$$
\begin{aligned}
& \Phi_{n+1}=\left\|t_{T_{n+1}}^{b}\right\|-\mu\left(\alpha_{n+1}\right) t_{N_{n+1}} \leqslant 0 \\
& \gamma_{n+1} \geqslant 0 \\
& \gamma_{n+1} \Phi_{n+1}=0
\end{aligned}
$$

Assuming that $\Phi_{n+1}^{\text {trial }}>0$, otherwise $\gamma_{n+1}=0$ and the trial state actually is the final state, the discrete consistency parameter $\gamma_{n+1}$ can be computed by enforcing the discrete counterpart of the consistency condition $\Phi_{n+1}=0$. Then, the frictional return mapping takes the form

$$
\begin{aligned}
& \boldsymbol{t}_{T_{n+1}}^{b}=\left(1-\epsilon_{T} \frac{\gamma_{n+1}}{\left\|t_{T_{n+1}^{\text {trial }}}^{b}\right\|}\right) t_{T_{n+1}^{\text {trial }}}^{b}=\mu\left(\alpha_{n+1}\right) t_{N_{n+1}} \\
& \alpha_{n+1}=\alpha_{n+1}^{\text {trial }}+\gamma_{n+1}\left[(1-w)+w\left\|t_{T_{n+1}^{\text {trial }}}^{b}\right\|-w \epsilon_{T} \gamma_{n+1}\right] \\
& \Phi_{n+1}:=\left\|t_{T_{n+1}^{\text {trial }}}^{b}\right\|-\epsilon_{T} \gamma_{n+1}-\mu\left(\alpha_{n+1}\right) t_{N_{n+1}}=0
\end{aligned}
$$

Computation of the consistency parameter $\gamma_{n+1}$ will require, in general, to solve the nonlinear equation $\Phi_{n+1}=\hat{\Phi}\left(\gamma_{n+1}\right)=0$, where it is implicitly understood that we are looking at $\alpha_{n+1}$ as a function $\alpha_{n+1}=$ $\alpha_{n+1}\left(\gamma_{n+1}\right)$, using $(3.7)_{2}$. Using a Newton-Raphson method the linearization of the slip function yields

$$
\Phi_{n+1}^{(k)}+D \Phi_{n+1}^{(k)} \cdot \Delta \gamma_{n+1}^{(k)}=0
$$

with

$\Phi_{n+1}^{(k)}=\Phi_{n+1}^{\text {trial }}-\epsilon_{T} \gamma_{n+1}^{(k)}-\left[\mu\left(\alpha_{n+1}^{(k)}\right)-\mu\left(\alpha_{n+1}^{\text {(rial }}\right)\right] t_{N_{n+1}}$
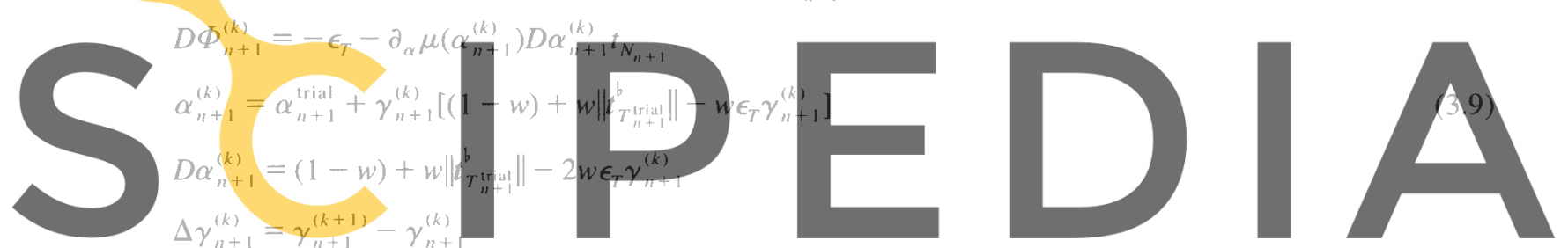

Register for free at https//Ww wh $w^{(0)}$.scipedia.com to download the version without the watermark

\subsubsection{Generalized PMP IRK method. The Generalized PMP IRK method is constructed via a two-stage} product formula algorithm as follows:

Stage 1 . A BE algorithm is applied to integrate the constrained evolution problem within a time sub-interval $\left[t_{n}, t_{n+\vartheta}\right] \subset\left[t_{n}, t_{n+1}\right]$ where $t_{n+\vartheta}:=(1-\vartheta) t_{n}+\vartheta t_{n+1}$ and $\vartheta \in(0,1]$. Thus, the first stage of the algorithm is identical to the scheme already described above. Explicitly, the following steps are performed for prescibed initial data $\left\{t_{T \alpha, n}{ }_{T<f}\right\}$ and given relative (parametrized) slip increment $\bar{g}_{T_{n+\vartheta}^{\alpha}}:=\bar{\xi}_{n+\vartheta}^{\alpha}-\bar{\xi}_{n}^{\alpha}$ :

Step 1. Define the generalized mid-point trial state according to

$$
\begin{aligned}
& t_{T_{\alpha, n+\vartheta}}^{\text {trial }}:=t_{T_{\alpha, n}}+\epsilon_{T} M_{\alpha \beta}\left(\bar{\xi}_{n+\vartheta}^{\beta}-\bar{\xi}_{n}^{\beta}\right) \\
& \alpha_{n+\vartheta}^{\text {trial }}:=\alpha_{n} \\
& \Phi_{n+\vartheta}^{\text {rial }}:=\left\|t_{T_{n+\vartheta}^{\text {rial }}}^{b}\right\|-\mu\left(\alpha_{n+\vartheta}^{\text {trial }}\right) \\
& t_{N_{n+\vartheta}}
\end{aligned}
$$

Step 2. The return mapping defines the final state at the generalized mid-point configuration $\mathscr{C}_{n+\vartheta}$ as the solution of the discrete constrained incremental algebraic problem:

$$
\begin{aligned}
& t_{T_{\alpha, n+\vartheta}}=t_{T_{\alpha, n+\vartheta}^{\text {trial }}}-\epsilon_{T} \gamma_{n+\vartheta} p_{T_{\alpha, n+\vartheta}} \\
& \alpha_{n+\vartheta}=\alpha_{n+\vartheta}^{\text {trial }}+\gamma_{n+\vartheta}\left[(1-w)+w\left\|t_{T_{n+\vartheta}}^{b}\right\|\right]
\end{aligned}
$$




$$
\begin{aligned}
& \Phi_{n+\vartheta}=\left\|t_{T_{n+\vartheta}}^{b}\right\|-\mu\left(\alpha_{n+\vartheta}\right) t_{N_{n+\vartheta}} \leqslant 0 \\
& \gamma_{n+\vartheta} \geqslant 0 \\
& \gamma_{n+\vartheta} \Phi_{n+\vartheta}=0
\end{aligned}
$$

Stage IIA. Since the trial values $t_{T_{\alpha, n+\vartheta}^{\text {trial }}}$ and the converged values $t_{T_{\alpha, n+\vartheta}}$ are available from Stage I and within the context of a product formula algorithm, the initial data $t_{T_{\alpha, n+\vartheta}}^{*}$ and $\alpha_{n+\vartheta}^{*}$ for the second stage are defined using the linear extrapolation:

$$
\begin{aligned}
& t_{T_{\alpha, n+\vartheta}}^{*}:=\frac{1}{\vartheta} t_{T_{\alpha, n+\vartheta}}-\frac{1-\vartheta}{\vartheta} t_{T_{T \alpha, n+\vartheta}} \\
& \alpha_{n+\vartheta}^{*}:=\frac{1}{\vartheta} \alpha_{n+\vartheta}-\frac{1-\vartheta}{\vartheta} \alpha_{n+\vartheta}^{\text {trial }}
\end{aligned}
$$

Within a finite deformation framework, all the objects involved in the linear extrapolation given by (3.13) should be viewed as objects lying in the same generalized mid-point configuration $C_{n+\vartheta}$. Thus, for the friction Coulomb model this extrapolation is performed on the plane $t_{N}=t_{N_{n+9}}$ of the tractions space.

Stage IIB. The second part of Stage II is identical to Stage I, where now the initial prescribed data becomes $t_{T^{*}+\vartheta}$ and the given (parametrized) relative slip increment is $\bar{g}_{T^{\alpha+1}}:=\bar{\xi}_{n+1}^{\alpha}-\bar{\xi}_{n+\vartheta}^{\alpha}$. The steps involved in the update are the following:

\section{Step 1. Define the trial state according to}
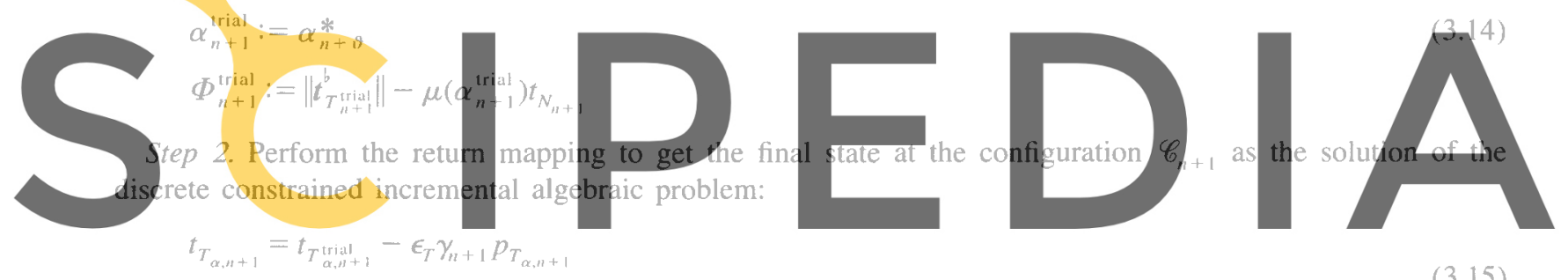

Register for free atiqnttps/fwww scipedia.f.com to download the version without the watermark

$$
\begin{aligned}
& \Phi_{n+1}=\left\|k_{T_{n+1}}^{b}\right\|-\mu\left(\alpha_{n+1}\right) i_{N_{n+1}} \leqslant 0 \\
& \gamma_{n+1} \geqslant 0 \\
& \gamma_{n+1} \Phi_{n+1}=0
\end{aligned}
$$

A rigorous stability and accuracy analysis of the two-stage, implicit, PMP algorithm, within the context of J2 plasticity, was provided by Simo [7]. The accuracy and stability analysis show that the generalized PMP algorithm is obviously consistent, second-order accurate for the PMP algorithm $(\vartheta=0.5)$, B-stable for $\vartheta \geqslant 0.5$ and ensures that the final stage is on the admissible domain. Remarkably, in sharp contrast with other second-order accurate algorithms, i.e. mid-point rule, second order accuracy is achieved performing a radial return mapping in each of the stages and thus a solution will be always guaranteed to exist for arbitrarily large time-steps. However, the long-term behaviour of this scheme is not optimal when compared with that exhibited by the, less accurate, BE algorithm. In contrast, this scheme becomes optimal for short-term behavior.

\subsubsection{Linearization of the frictional time-stepping algorithm}

The frictional time-stepping algorithms presented above are amenable to exact linearization, leading to the corresponding terms of the consistent or algorithmic tangent operator. In order to accommodate the linearization of the BE and PMP return mapping algorithms into a single expression, the linearization of the frictional traction at time $t_{n+\vartheta}$, can be derived at the generic configuration $\mathscr{C}_{n+\vartheta}$, where $\vartheta=1$ for the BE algorithm and $\vartheta \in(0,1]$ for the PMP algorithm. We point out that the implementation of the PMP IRK algorithm actually requires only 
the linearization of the Stage I, while Stage II can be viewed as an update procedure to provide the initial conditions for the next time step, after convergence has been achieved.

Using the directional derivative, the linearization of the frictional time integration algorithm leads to the following expressions.

Step 1: Trial state. The linearization of the trial state takes the form

$$
\begin{aligned}
& \Delta t_{T_{\alpha, n+\vartheta}}:=\Delta t_{T_{\alpha, n+\vartheta}^{\text {trial }}} \\
& \Delta \alpha_{n+\vartheta}:=\Delta \alpha_{n+\vartheta}^{\text {trial }}=0
\end{aligned}
$$

Step 2: Return mapping. The linearization of the return mapping takes the form

$$
\begin{aligned}
& \Delta t_{T_{\alpha, n+\vartheta}}:=\mu\left(\alpha_{n+\vartheta}\right) \Delta t_{N_{n+\vartheta}} p_{T_{\alpha, n+\vartheta}^{\text {trial }}}+\mu\left(\alpha_{n+\vartheta}\right) t_{N_{n+\vartheta}} \Delta p_{T_{\alpha, n+\vartheta}^{\text {trial }}}+\partial_{\alpha} \mu\left(\alpha_{n+\vartheta}\right) \Delta \alpha_{n+\vartheta} t_{N_{n+\vartheta}} p_{T_{\alpha, n+\vartheta}^{\text {trial }}} \\
& \Delta \alpha_{n+\vartheta}:=\Delta \gamma_{n+\vartheta}\left[(1-w)+w \mu\left(\alpha_{n+\vartheta}\right) t_{N_{n+\vartheta}}-w \epsilon_{T} \gamma_{n+\vartheta}\right]+\gamma_{n+\vartheta} w \Delta\left\|t_{T_{n+\vartheta}^{\text {trial }}}^{b}\right\|
\end{aligned}
$$

with

$$
\begin{aligned}
& \Delta t_{N_{n+\vartheta}}:=\epsilon_{N} H\left(g_{N_{n+\vartheta}}\right) \Delta g_{N_{n+\vartheta}} \\
& \Delta t_{T_{\alpha, n+\vartheta}^{\text {triat }}}:=\Xi_{\alpha \beta} \Delta \bar{\xi}_{n+\vartheta}^{\beta} \\
& \Delta \gamma_{n+\vartheta}:=\frac{1}{\epsilon_{T}}\left[\Delta\left\|t_{T_{n+\vartheta}^{\text {triat }}}^{b}\right\|-\mu\left(\alpha_{n+\vartheta}\right) \Delta t_{N_{n+\vartheta}}\right]-\frac{1}{\epsilon_{T}} \partial_{\alpha} \mu\left(\alpha_{n+\vartheta}\right) t_{N_{n+\vartheta}} \Delta \alpha_{n+\vartheta}
\end{aligned}
$$

where $H(\cdot)$ is the Heaviside function and

$$
\begin{aligned}
& \Xi_{\alpha \beta}:=\epsilon_{T}\left(M_{\alpha \beta}+M_{\alpha \gamma, \beta} g_{T}^{\gamma}\right) \\
& g_{T}^{\alpha}:=\bar{\xi}_{n+\vartheta}^{\alpha}-\bar{\xi}_{n}^{\alpha}
\end{aligned}
$$

with the, in general, non-symmetric operator $\Xi_{\alpha \beta}$ evaluated at $t_{n+\vartheta}$.

Introducing $\Delta \gamma_{n+\vartheta}$ into the expression of $\Delta \alpha_{n+\vartheta}$ and collecting terms, leads to

$$
\Delta \alpha_{n+\vartheta}:=\beta_{1} \Delta\left\|\boldsymbol{t}_{T_{n+\vartheta}^{\text {trial }}}^{\text {tial }}\right\|-\beta_{2} \mu\left(\alpha_{n+\vartheta}\right) \Delta t_{N_{n+\vartheta}}
$$

with

$$
\begin{aligned}
& \beta_{1}:=\frac{\partial \alpha /\left.\partial \gamma\right|_{n+\vartheta}+w \epsilon_{T} \gamma_{n+\vartheta}}{\epsilon_{T}+\partial \alpha /\left.\partial \gamma\right|_{n+\vartheta} \partial_{\alpha} \mu\left(\alpha_{n+\vartheta}\right) t_{N_{n+\vartheta}}} \\
& \beta_{2}:=\frac{\partial \alpha /\left.\partial \gamma\right|_{n+\vartheta}}{\epsilon_{T}+\partial \alpha /\left.\partial \gamma\right|_{n+\vartheta} \partial_{\alpha} \mu\left(\alpha_{n+\vartheta}\right) t_{N_{n+\vartheta}}}
\end{aligned}
$$

where

$$
\partial \alpha /\left.\partial \gamma\right|_{n+\vartheta}:=(1-w)+w \mu\left(\alpha_{n+\vartheta}\right) t_{N_{n+\vartheta}}-w \epsilon_{T} \gamma_{n+\vartheta}
$$

\section{Tool wear mechanisms and wear estimate}

Wear phenomena are the dominanting failure mechanism of dies in both sheet and bulk metal forming operations. When considering wear in forming processes, attention should be focussed on the following items: type of die failure, choice of die material, surface treatment and lubrication, process parameters such as temperature and forming speed and predominant wear mechanisms. The life-time of a die is usually expressed by the number of parts that can be produced before the dimensions of the parts exceed the given tolerances or serious damage of the die occur. To be able to estimate, to predict and to incorporate into a numerical model the complex wear phenomena will improve considerably the life of dies in metal forming operations. 


\subsection{Wear mechanisms in hot forging processes}

Statistical results show that wear is up to a $60-70 \%$, the dominating failure mechanism for hot forging dies, and has therefore an important influence on the production costs of forged products. Critical regions in a forging die are places exposed to a very high pressure, internal corners with a notch effect, areas that reach a very high temperature and finally regions with large slip amounts on the die. Statistical investigations of the type of die failure which have the greatest influence on the scrapping of forging dies, show that the primary reason for scrapping a die are wear phenomena at external corners or at roundings. In order to describe wear as the main reason for the scrapping of forging tools it is important to determine which are the main mechanisms that causes wear. It is generally agreed that the most important wear mechanism in hot forging is three body abrasive wear caused by hard scale particles embedded in the surface of the work piece. Experimental results show that the amount and type of scale, the adhesion of the scale to the surface of the work piece and the hardness (chemical structure) of the scale are the determining factors for tool wear. Wear is also found to be proportional to the hardness of the work piece material and inversely proportional to the hardness of the die material at the maximum temperature reached by the surface during the forging process [20].

\subsection{Wear mechanisms in sheet metal forming processes}

In sheet metal forming processes, it has been found that $65 \%$ of tool failure is caused by adhesive and abrasive wear in the drawbead and die radius regions [21]. Due to the surface roughness and asperities, when two surfaces are pressed together, the real contact area will be much smaller than the apparent one. The pressure on these asperities will be sufficiently high to cause plastic deformations on the asperities. Also the sliding of the sheet over the tool surface leads to heating due to frictional dissipation. The high surface pressure combined with the heat generation due to frictional dissipation leads to welding of the asperities of the tool and sheet surfaces. The break off of these welded asperities can scratch the tool surface. Investigations results on failure frequency due to surface damage in sheet metal forming tools and the influence of tool and sheet hardness, lubricant, blankholder pressure and sliding distance on wear in sheet metal forming can be found in [21].

\subsection{Wear estimate}

The two most important mechanisms of wear in forming processes have been identified as adhesive and abrasive wear.

\subsubsection{Adhesive wear}

During relative sliding between two surfaces, the lubricant film may thin out and break down, allowing the two materials to cold weld at the asperities contact. Further relative sliding will break either in the cold welded contact or in one of the materials. This process does not produce any free wear particles but could possibly transfer a small amount of material from one surface to another. On the other hand, by continuous sliding a free wear particle can be formed by two mechanisms: (a) Adhesion of particles takes place under high pressure. Continuous sliding relieves the pressure in the contact and the particle may break off. (b) Due to chemical changes in the particle when it is transferred from one surface to another, the particle may oxidize and thus adhere poorly to the new surface. The free wear particles and the accumulation of material formed by adhesive wear will often cause abrasive wear.

Assuming circular contacting asperities of the same size, Archard [15] proposed a model for adhesive wear with the following assumptions: it is proportional to the local sliding length and to the normal pressure, it is inversely proportional to the local hardness of the surface (the yield stress of a deformed asperity) and it is dependent on a wear constant (to be determined experimentally and ranging from 0 to 1 ), which indicates the probability of the formation of a particle in an asperity contact. This constant will depend on the work piece and die material, the tendency to cold weld, and the interface conditions (surface films, lubricant film, temperature, etc.). Then Archard's law for adhesive wear can be written (in rate form) as

$$
\dot{Z}=K_{\mathrm{adh}}[q \dot{s} / H]
$$


where $Z$ is the (adhesive) wear volume per unit area, $q$ is the local normal pressure, $s$ is the local sliding length, $H$ is the local hardness of the material, $K_{\text {adh }}$ is the adhesive wear constant and the superposed dot means material time derivation. Typical values of $K_{\text {adh }}$ based on continuum mechanical and metallurgical investigations for different material combinations can be found in [22]. The experimental determination of $K_{\text {adh }}$ is difficult and subject to some uncertainty. Many experimental investigations of wear confirm the validity of Archard's wear law, especially for relatively small contact normal pressures [9]. Some investigations show that Archard's law significantly underestimates the wear when the normal pressure exceeds the yield stress of the material [9].

\subsubsection{Abrasive wear}

The term abrasive covers the situations of two-body and three-body abrasive wear. In two-body abrasive wear an asperity from the harder material ploughs a furrow in the softer material during the relative sliding. In three-body abrasive wear the furrow is ploughed by a hard particle. In two-body abrasive wear, normally only the softer surface is subjected to wear and if the harder surface is sufficiently smooth, it is possible to eliminate the two-body abrasive wear totally. The three-body abrasive wear is much more difficult to eliminate as it is very difficult to avoid impurities, including hard particles, between the two surfaces in contact. Hard particles that can cause abrasion are, for instance, dust, wear particles from adhesive wear that are very hard after severe plastic deformations, particles formed by corrosion of the surface (i.e. scale in hot forging), etc.

Assuming a number of isolated and uniform asperity contacts, it is possible to derive a simplified model for abrasive wear, similar to the Archard's model for adhesive wear [9]. Then the law for abrasive wear can be written (in rate form) as

$$
\dot{Z}=K_{\text {abr }}[q \dot{s} / H]
$$

where $Z$ is the (abrasive) wear volume per unit area and $K_{\mathrm{abr}}$ is the abrasive wear constant. The abrasive wear law states that (instantaneous) abrasive wear is proportional to the local sliding length and normal pressure, inversely proportional to the local hardness of the surface and depends of an abrasive wear constant (to be determined experimentally and ranging from 0 to 1 ), now dependent on the surface topography, the presence of hard abrasive particles, lubricant, etc.

From (4.1) and (4.2) a unified law for adhesive and abrasive wear [9] can be written (in rate form) as

$$
\dot{Z}=K_{\text {wear }}[q \dot{s} / H]
$$

where now $Z$ is the (adhesive/abrasive) wear volume per unit area and $K_{\text {wear }}$ is a wear constant to be determined experimentally (ranging from 0 to 1 ), which for adhesive wear mechanisms will depend mainly on the material combination, interface conditions, lubricant and temperature, and for abrasive wear mechanisms will depend mainly on surface topology, hard particles between surfaces and lubricant. Wear models based in (4.3) have been used in computational codes by Owen et al. [11], de Souza Neto et al. [12], Stromberg et al. [13] and Stromberg [14], among others.

The local hardness of the die surface is a strong function of the local temperature of the die surface and a fully coupled thermomechanical model would be necessary. The sliding length, which is the amount of material passing a specific point on the die surface, is considered to be the most important parameter in the wear estimation, due to its strong influence on the heat generation by frictional dissipation.

In the time discrete setting, within a typical time sub-interval $\left[t_{n}, t_{n+1}\right] \subset[0, T]$ of the time interval of interest and using for instance a $\mathrm{BE}$ algorithm, time integration of wear volume rate per unit area given by (2.3), leads to the discrete adhesive and abrasive wear algorithmic expression

$$
Z_{n+1}=Z_{n}+K_{\text {wear }}\left[q_{n+1}\left(s_{n+1}-s_{n}\right) / H_{n+1}\right]
$$

where $(\cdot)_{n}$ and $(\cdot)_{n+1}$ denote the algorithmic approximation to their exact values at times $t_{n}$ and $t_{n+1}$, respectively. 
REMARK 2.1. Under some simplified assumptions, i.e. constant surface material hardness, the wear evolution estimate can be integrated in closed-form leading to an explicit wear function of the (accumulated) frictional dissipation.

Let us consider, as a simple model problem, a Coulomb frictional model with a friction coefficient defined as a function of the frictional dissipation rate. Then, the frictional dissipation evolution equation takes the form:

$$
\mathscr{D}_{\text {fric }}:=\dot{\alpha}:=\mu(\alpha) q \dot{s}
$$

Using (4.3) and (4.5) the following expression can be derived

$$
\dot{Z}=\frac{K_{\text {wear }}}{H} \frac{\dot{\alpha}}{\mu(\alpha)}
$$

Let us consider now two simple cases: friction coefficient as a linear function of frictional dissipation and constant friction coefficient.

(i) Linear friction coefficient. Assume a friction coefficient described by a linear function of the frictional dissipation

$$
\mu(\alpha):=\mu_{0}+\mu_{1} \alpha
$$

Substituting (4.7) into (4.6) and integrating leads to the following logarithmic closed-form expression for the wear estimate

$$
Z=\frac{K_{\text {wear }}}{\mu_{1} H} \log \left[\frac{\mu(\alpha)}{\mu_{0}}\right]
$$

(ii) Constant friction coefficient. Assume a constant friction coefficient

$$
\mu(\alpha):=\mu_{0}
$$

Substituting (4.9) into (4.6) and integrating leads to the following linear closed-form expression for the wear estimate

$$
Z=\frac{K_{\text {wear }}}{\mu_{0} H} \alpha
$$

\section{Numerical simulations}

The formulation presented in the preceding sections is illustrated below in a number of numerical simulations. The goals are to provide a practical accuracy assessment of the frictional wear model and to demonstrate the robustness of the overall frictional contact formulation in different numerical simulations and particularly in metal forming operations. The calculations are performed with an enhanced version of the finite element program FEAP developed by R.L. Taylor and J.C. Simo and documented in [16].

\subsection{Draw Bead Simulator}

This example is concerned with the simulation of a draw bead in a deep drawing sheet metal forming process. An initially flat GA sheet metal strip will be drawn through a set of rollers. The material properties for the GA sheet metal strip were taken as bulk modulus $K=171.6 \mathrm{GPa}$, shear modulus $G=79.2 \mathrm{GPa}$ and a power hardening law given by the Swift equation

$$
\sigma_{Y}=536.0\left(0.0033+\bar{\varepsilon}^{p}\right)^{0.21} \mathrm{MPa}
$$




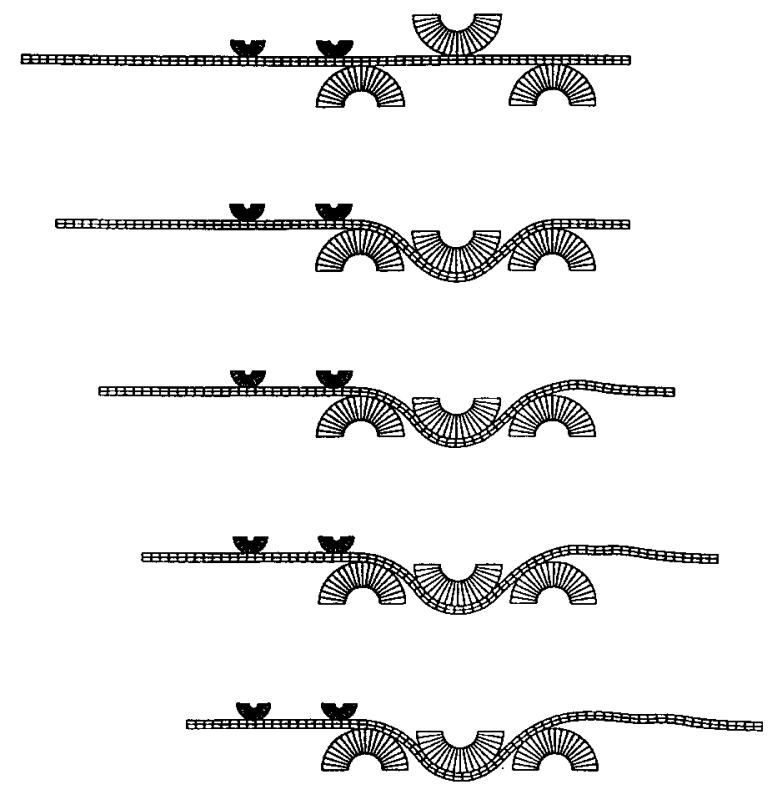

Fig. 3. Draw Bead Simulator. Initial configuration and deformed shapes of the strip at five different stages of the process, corresponding to a displacement of the upper main roller of $6.350 \mathrm{~mm}$, at the end of the first phase, and to prescribed displacements of $5,10 \mathrm{and} 15 \mathrm{~mm}$ of the right edge of the strip, during the second phase, respectively.

The rollers were considered as rigid surfaces. Frictional wear phenomena at the interfaces, between the GA metal sheet and the rigid rollers, was modeled using a frictional softening curve defined in terms of the frictional dissipation $\alpha$ as

$$
\mu=0.078-0.666 \times 10^{-2} \alpha
$$

where $\alpha$ is measured in $\mathrm{KN} / \mathrm{cm}$. The strip was $1 \mathrm{~mm}$ thick and measured $70 \mathrm{~mm}$ length. The three main rollers and the two guide rollers were of radius 5 and $2 \mathrm{~mm}$, respectively. The separation between the three main rollers was $11 \mathrm{~mm}$ and the upper main roller was positioned at a distance of $20 \mathrm{~mm}$ from the right edge of the strip. The distance between the upper main roller and the two guide rollers was 14 and $24 \mathrm{~mm}$, respectively. Fig. 3 shows the initial geometry of the test. Plane strain conditions have been assumed and only a half part of each roller has been discretized. The loading process consists of two phases. In the first phase, the main upper roller goes down up to a distance of $6.35 \mathrm{~mm}$ while the right edge of the strip is kept fixed, creating a situation of three point bending load. This first phase simulates the clamping of the sheet by the blankholder at the beginning of a deep drawing process. In the second phase, the rollers are kept fixed and the strip is pulled out from the right edge up to a final distance of $15 \mathrm{~mm}$.

The geometry of the problem was modeled with 140 continuum elements being utilized for the discretization

Table 1

Draw bead simulator. Euclidean norm of the residual for four typical time steps

\begin{tabular}{llll}
\hline Step 20 & Step 70 & Step 120 & Step 170 \\
\hline $3.94977 \mathrm{E}+05$ & $1.88688 \mathrm{E}+07$ & $1.88642 \mathrm{E}+07$ & $1.88639 \mathrm{E}+07$ \\
$3.16066 \mathrm{E}+06$ & $2.23098 \mathrm{E}+05$ & $2.22340 \mathrm{E}+05$ & $2.18650 \mathrm{E}+05$ \\
$2.37233 \mathrm{E}+05$ & $2.47160 \mathrm{E}+04$ & $4.21119 \mathrm{E}+04$ & $4.36364 \mathrm{E}+04$ \\
$1.80076 \mathrm{E}+04$ & $2.83830 \mathrm{E}+04$ & $3.14779 \mathrm{E}+03$ & $1.27160 \mathrm{E}+04$ \\
$2.44798 \mathrm{E}+03$ & $1.88422 \mathrm{E}+03$ & $1.91413 \mathrm{E}+03$ & $1.08463 \mathrm{E}+02$ \\
$8.78457 \mathrm{E}+00$ & $1.75791 \mathrm{E}+02$ & $8.94470 \mathrm{E}+02$ & $8.30284 \mathrm{E}-02$ \\
$2.85522 \mathrm{E}-04$ & $4.30222 \mathrm{E}-01$ & $2.67843 \mathrm{E}+02$ & $5.91113 \mathrm{E}-06$ \\
& $3.41143 \mathrm{E}-06$ & $2.85655 \mathrm{E}-01$ & \\
\hline
\end{tabular}


of the strip, using 2 elements across the thickness, and 20 elements being used for the discretization of each of the rollers. A mixed Q1/P0 finite element formulation at finite strains was used for the discretization of the strip. Frictional contact constraints were regularized by means of penalty method and the normal and tangential penalty parameters were taken as $\varepsilon_{N}=5 \cdot 10^{11} \mathrm{~N} / \mathrm{m}^{3}$ and $\varepsilon_{T}=1 \cdot 10^{10} \mathrm{~N} / \mathrm{m}^{3}$, respectively. The loading process was achieved in 170 time steps, 20 steps for the first phase and 150 time steps for the second phase, through displacement control of the upper main roller and the right edge of the strip. The Newton-Raphson method, combined with a line search optimization procedure, was used to solve the nonlinear system of equations arising from the spatial and temporal discretization of the weak form of the momentum balance equation. Convergence
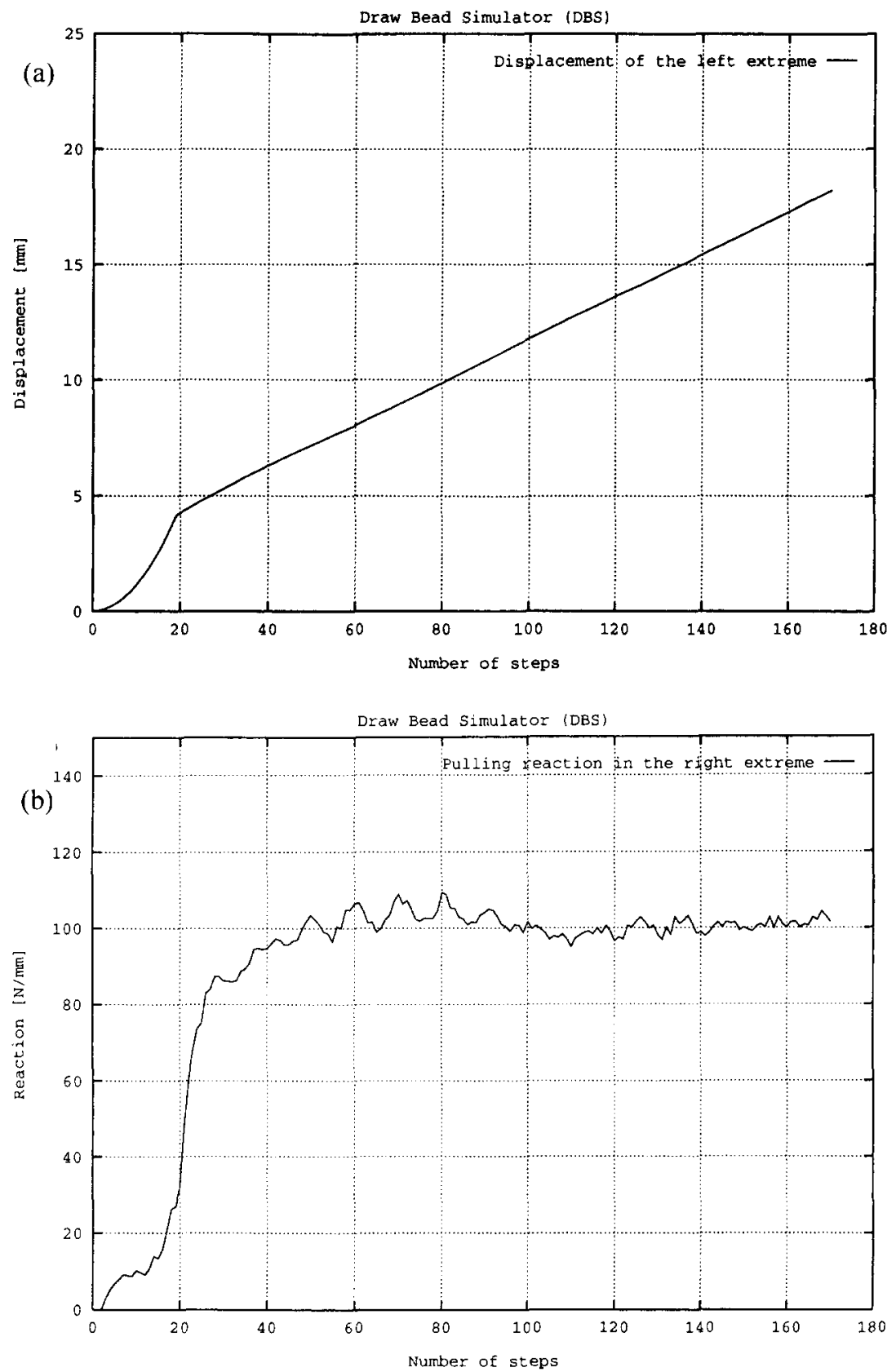

Fig. 4. Draw Bead Simulator. Evolution of the horizontal displacement at the left edge of the strip and the horizontal reaction at the right edge of the strip, during the loading process. 
of the incremental iterative solution procedure was monitored by requiring a tolerance of $10^{-18}$ in the energy norm.

The analysis was performed on a Silicon Graphics Power Challenge $L$ Workstation and it was accomplished in 34 min CPU time. Table 1 shows the Euclidean norm of the residual at four typical time steps.

Fig. 3 shows the initial geometry and deformed shapes of the strip at different stages of the process, corresponding to the end of the first phase, for a vertical displacement of $6.35 \mathrm{~mm}$ of the upper main roller, and to different prescribed displacements of 5,10 and 15 of the right edge of the strip, during the second phase.

Fig. 4 shows the evolution of the horizontal displacement of the left edge of the strip and the horizontal reaction at the right edge of the strip, during the loading process. Fig. 5 shows the wear profiles on the main rollers at the same selected stages of the analysis of Fig. 3 .
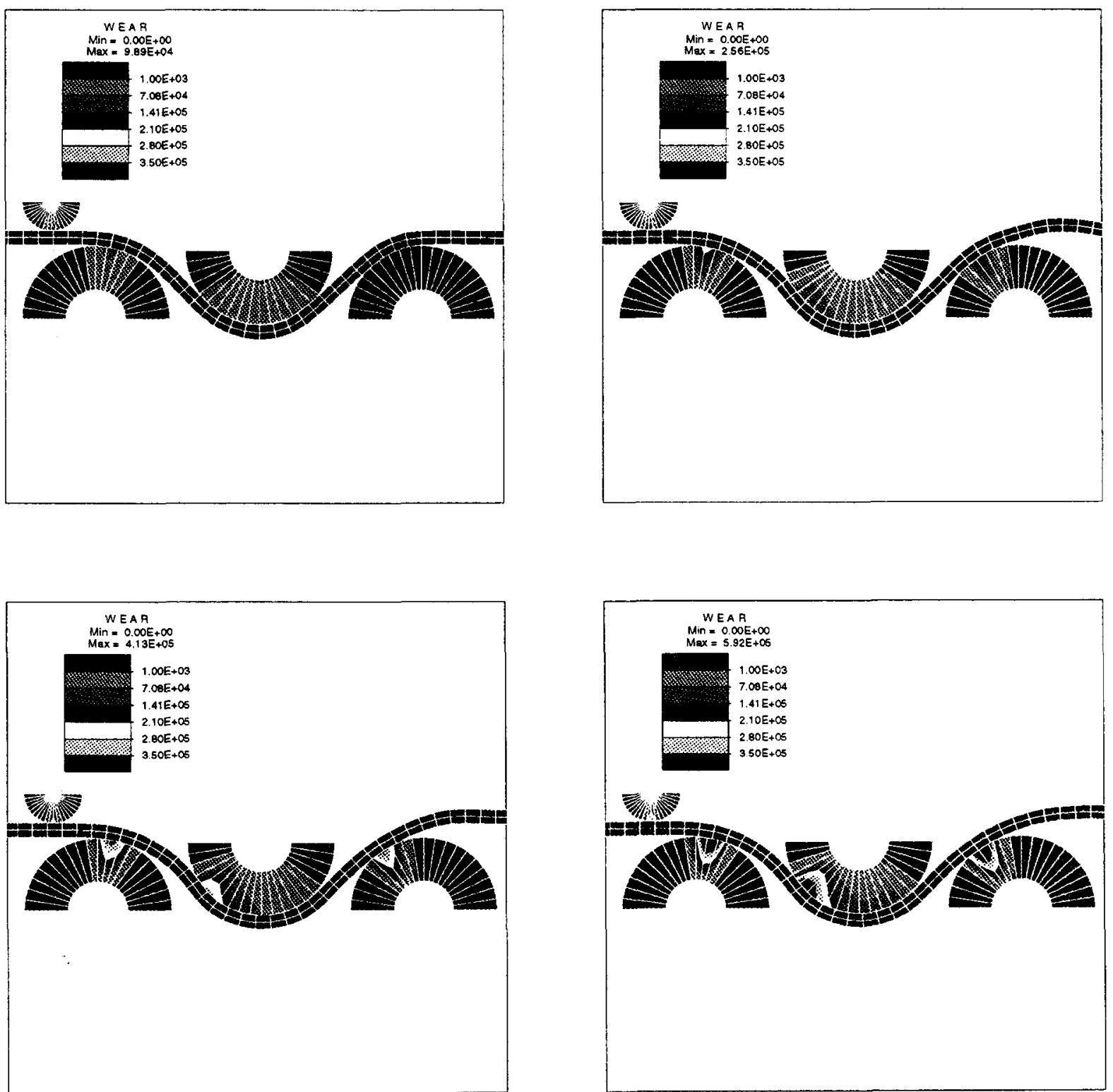

Fig. 5. Draw Bead Simulator. Wear profiles on the main rollers at different stages of the analysis. (a) At the end of the first phase for an upper main roller displacement of $6.350 \mathrm{~mm}$. (b) For a right edge prescribed horizontal displacement of $5 \mathrm{~mm}$, (c) $10 \mathrm{~mm}$ and (d) $15 \mathrm{~mm}$, during the second phase. 

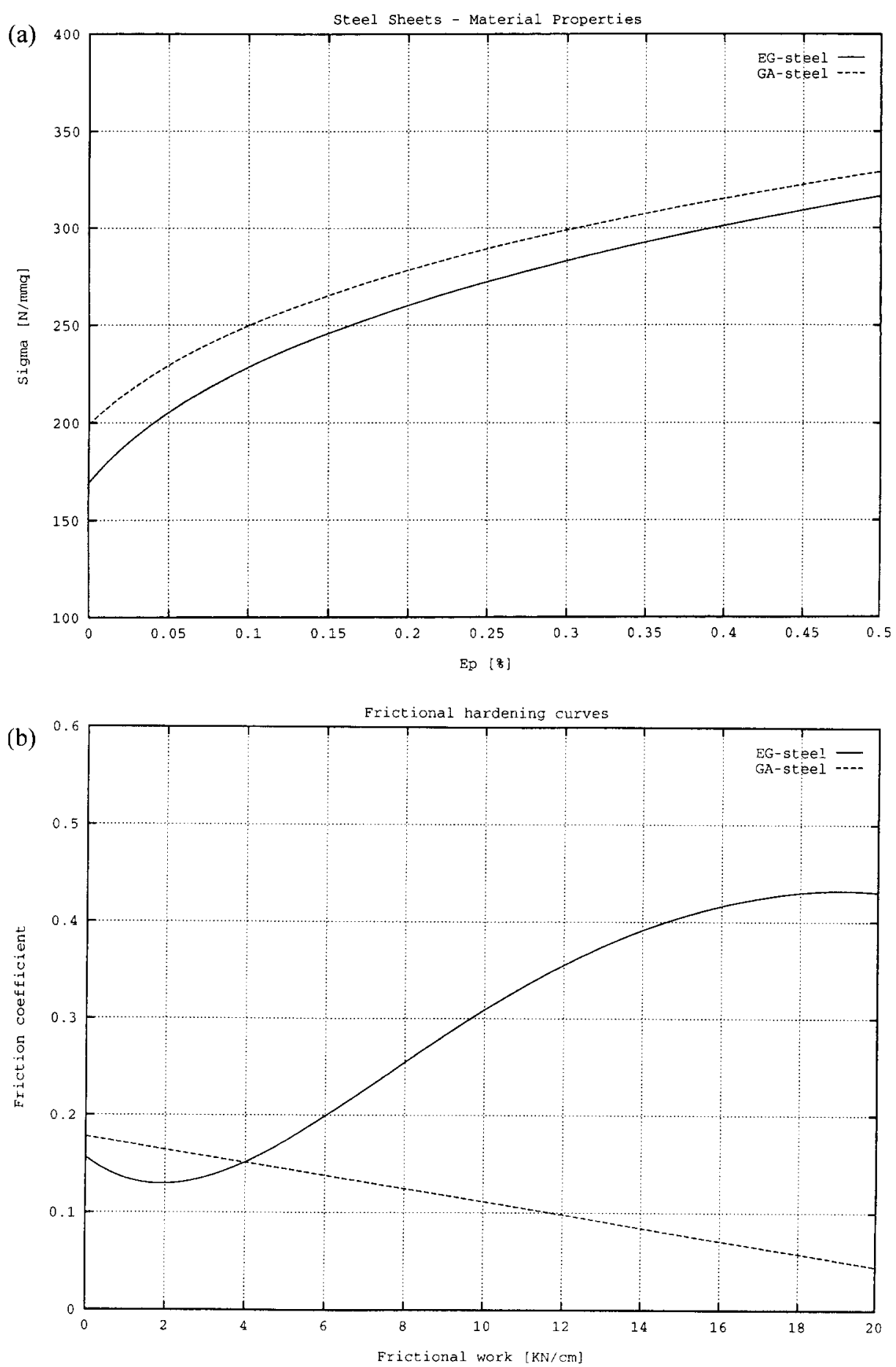

Fig. 6. Flat Sheet Sliding tests. (a) Hardening plasticity laws for the GA and EG steel materials. (b) Frictional hardening laws for the GA and EG steel materials.

Table 2

Flat Sheet Sliding tests. Mechanical properties for the GA and EG steel sheets

\begin{tabular}{lllllll}
\hline Material & $K(\mathrm{GPa})$ & $G(\mathrm{GPa})$ & $Y P(\mathrm{MPa})$ & $T S(\mathrm{MPa})$ & $E L(\%)$ & $n$ \\
\hline GA & 171.6 & 79.2 & 199.1 & 319.3 & 42.7 & 0.216 \\
EG & 171.6 & 79.2 & 169.2 & 310.0 & 45.4 & 0.243 \\
\hline
\end{tabular}


Table 3

Flat Sheet Sliding tests. Frictional hardening law for the GA and EG steel sheets. Coefficients of the polynomial function for frictional dissipation measured in $\mathrm{KN} / \mathrm{cm}$

\begin{tabular}{lllllll}
\hline Material & $a_{0}$ & $a_{1}$ & $a_{2}$ & $a_{3}$ & $a_{4}$ & $a_{5}$ \\
\hline GA & 0.178 & $-0.666 \times 10^{-2}$ & & & & \\
EG & 0.157 & $-0.315 \times 10^{-1}$ & $0.104 \times 10^{-1}$ & $-0.821 \times 10^{-3}$ & $0.289 \times 10^{-4}$ & $-0.410 \times 10^{-6}$ \\
\hline
\end{tabular}

\subsection{Flat Sheet Sliding tests}

This example is taken from de Souza Neto et al. [12] and is concerned with the numerical simulation of flat sheet sliding tests. The experimental tests are as follows. A steel flat sheet is clamped to the sliding table. A prescribed normal force is then applied to the tip of the tool material (SKD-11). The tip is kept fixed during the experiment to avoid rotation and ensure high precision in the measurement of the friction coefficient. Once the normal force has been applied, the table slides $300 \mathrm{~mm}$ driven by a hydraulic cylinder. After sliding, the normal force is released and the table returns to its initial position. The normal force is then reapplied and the cycle is repeated a number of times.

Two zinc coated sheet metals, typically employed in the manufacture of automotive body shells, have been considered: GA and EG steel sheets. The mechanical properties for the GA and EG steel sheets are shown in Table 2, where $K$ is the bulk modulus, $G$ is the shear modulus, $Y P$ is the initial yield stress, $T S$ is the maximum tensile strength, $E L$ is the elongation at rupture and $n$ is the exponent of the power law hardening for plasticity. Fig. 6 shows the hardening power law curves for the GA and EG steel sheets:
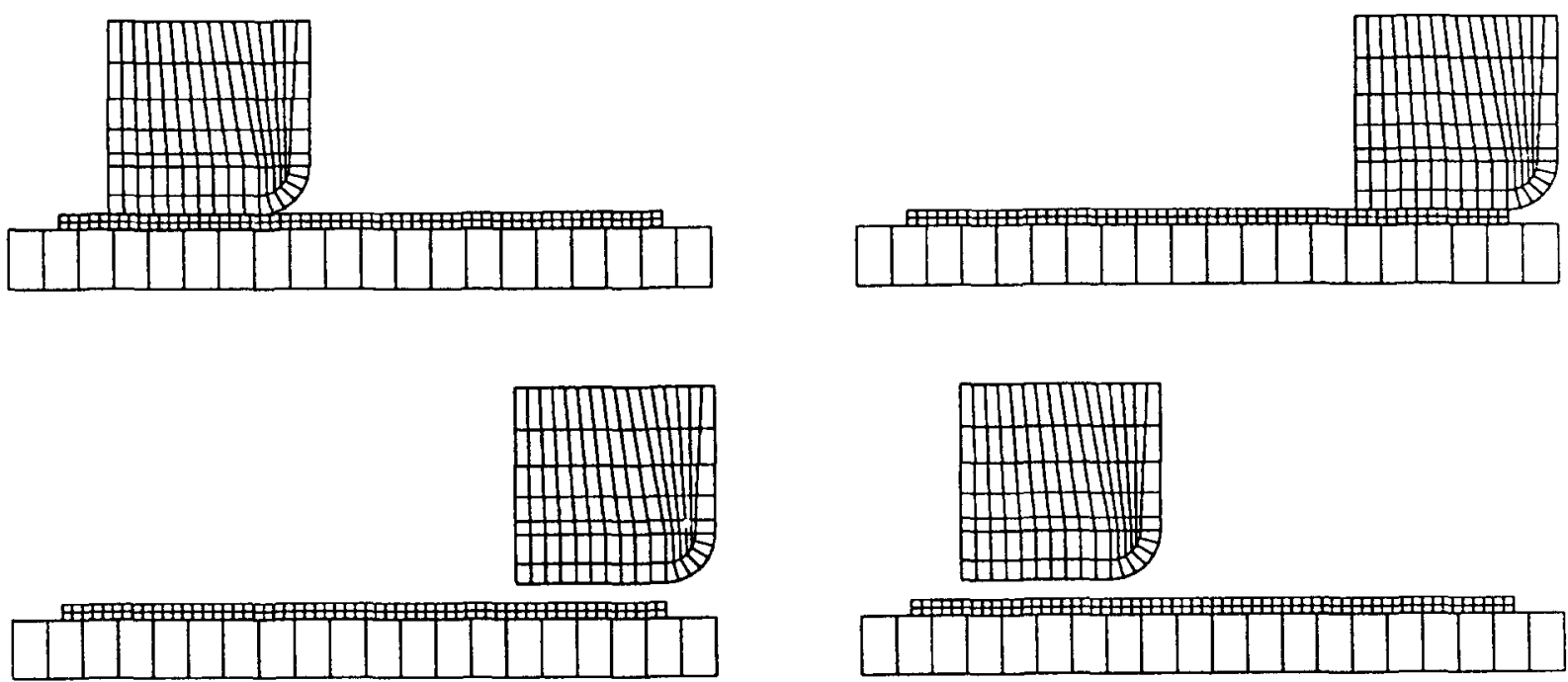

Fig. 7. Flat Sheet Sliding tests. Finite element mesh and sliding cycle. (a) Initial configuration and application of normal force; (b) sliding; (c) release normal force, and (d) return to initial configuration.

Table 4

Flat Sheet Sliding tests. Euclidean norm of the residual for four typical time steps. GA steel sheet. Normal force of $0.98 \mathrm{KN}$

\begin{tabular}{llll}
\hline Pass I & Pass 5 & Pass 10 & Pass 15 \\
\hline $1.23075 \mathrm{E}+09$ & $1.23075 \mathrm{E}+09$ & $1.23075 \mathrm{E}+09$ & $1.23075 \mathrm{E}+09$ \\
$4.70945 \mathrm{E}+04$ & $4.70696 \mathrm{E}+04$ & $4.70458 \mathrm{E}+04$ & $4.70230 \mathrm{E}+04$ \\
$1.23677 \mathrm{E}+04$ & $1.23583 \mathrm{E}+04$ & $1.23494 \mathrm{E}+04$ & $1.23408 \mathrm{E}+04$ \\
$8.04745 \mathrm{E}+02$ & $8.01015 \mathrm{E}+02$ & $7.97387 \mathrm{E}+02$ & $7.93858 \mathrm{E}+02$ \\
$1.21043 \mathrm{E}+02$ & $1.22529 \mathrm{E}+02$ & $1.23987 \mathrm{E}+02$ & $1.25417 \mathrm{E}+02$ \\
$2.20370 \mathrm{E}+00$ & $2.26997 \mathrm{E}+00$ & $2.33562 \mathrm{E}+00$ & $2.40061 \mathrm{E}+00$ \\
$7.13569 \mathrm{E}-06$ & $6.83974 \mathrm{E}-06$ & $6.70814 \mathrm{E}-06$ & $8.21763 \mathrm{E}-06$ \\
\hline
\end{tabular}




$$
\begin{aligned}
& \sigma_{Y}=374.0 \cdot\left(0.054+\bar{\varepsilon}^{\prime}\right)^{0.216} \text { MPa for the GA steel } \\
& \sigma_{Y}=367.6 \cdot\left(0.041+\bar{\varepsilon}^{\prime \prime}\right)^{0.24 .3} \text { MPa for the EG steel }
\end{aligned}
$$

Frictional behavior was modeled as a polynomial function of the frictional dissipation $\alpha$ given by

$$
\mu(\alpha)=a_{0}+a_{1} \alpha+a_{2} \alpha^{2}+\cdots+a_{p} \alpha^{p}
$$

The presence of a hard surface coating, difficult to remove, in the GA steel sheet leads to a progressive softening
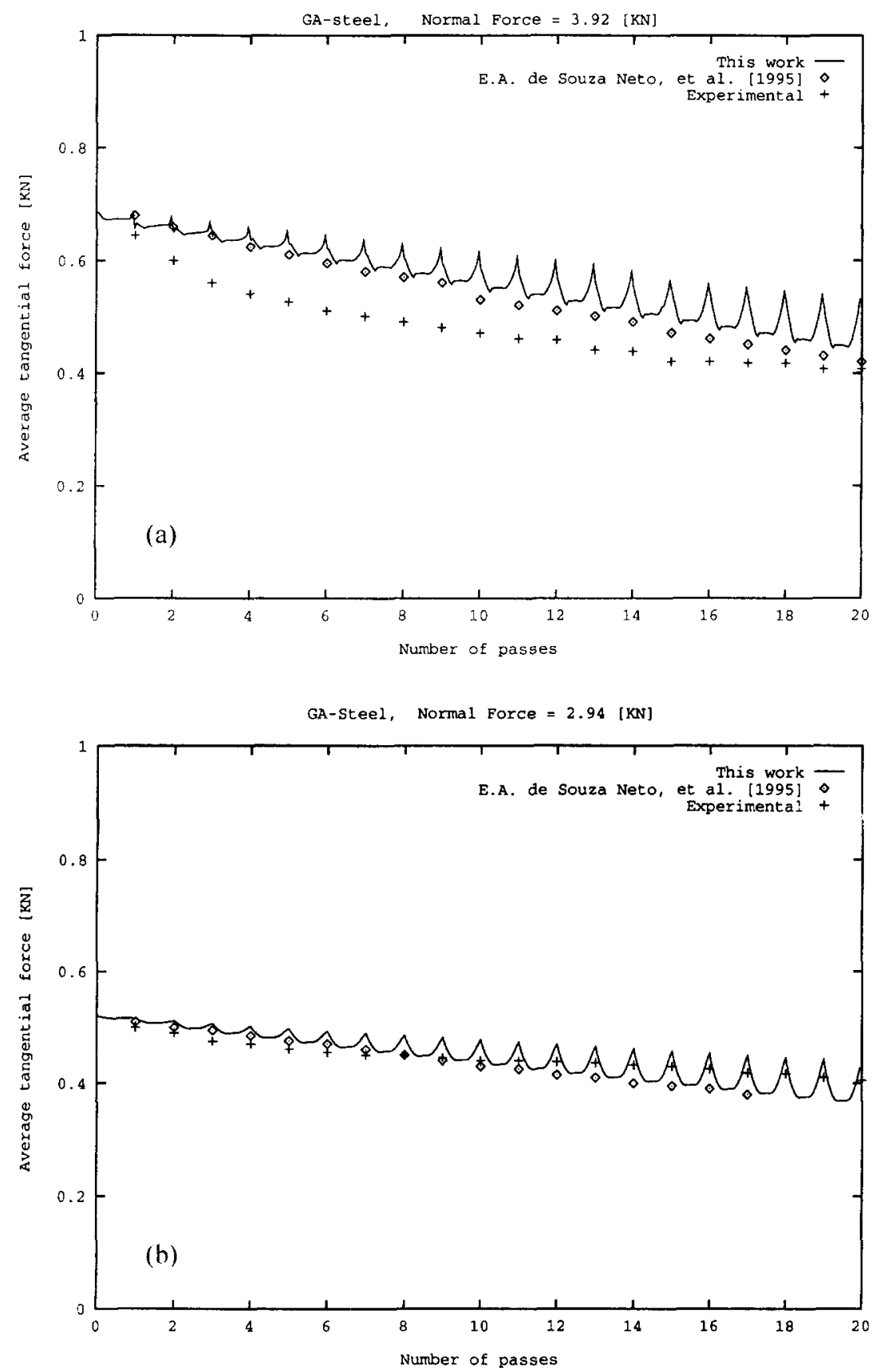

Fig. 8. Flat Sheet Sliding tests. Tangential force versus number of passes during the sliding tests using a GA steel sheet at different constant normal forces: (a) Normal force $=3.92 \mathrm{KN}$ : (b) Normal force $=2.94 \mathrm{KN}$. 
of the frictional behavior. In contrast with this behavior, the EG steel sheet experiences an initial softening, due to flattening of microasperities, followed by a substantial increase of the friction coefficient, due to the removal of its relatively soft zinc coat. The coefficients of the frictional hardening law, for a frictional dissipation $\alpha$ measured in $\mathrm{KN} / \mathrm{mm}$, for the GA and EG steel sheets are shown in Table 3. Frictional hardening behavior for the GA and EG steel sheets is shown in Fig. 6.

The sheet initially measured $400 \mathrm{~mm}$ long, $100 \mathrm{~mm}$ wide and $0.8 \mathrm{~mm}$ thick. The tip of the tool measured $10 \mathrm{~mm}$ long and $10 \mathrm{~mm}$ wide, with an inner radius of $2.5 \mathrm{~mm}$ at the bottom corner of the right edge. Then the tested surface at the experiment measured $300 \mathrm{~mm}$ long and $10 \mathrm{~mm}$ wide.
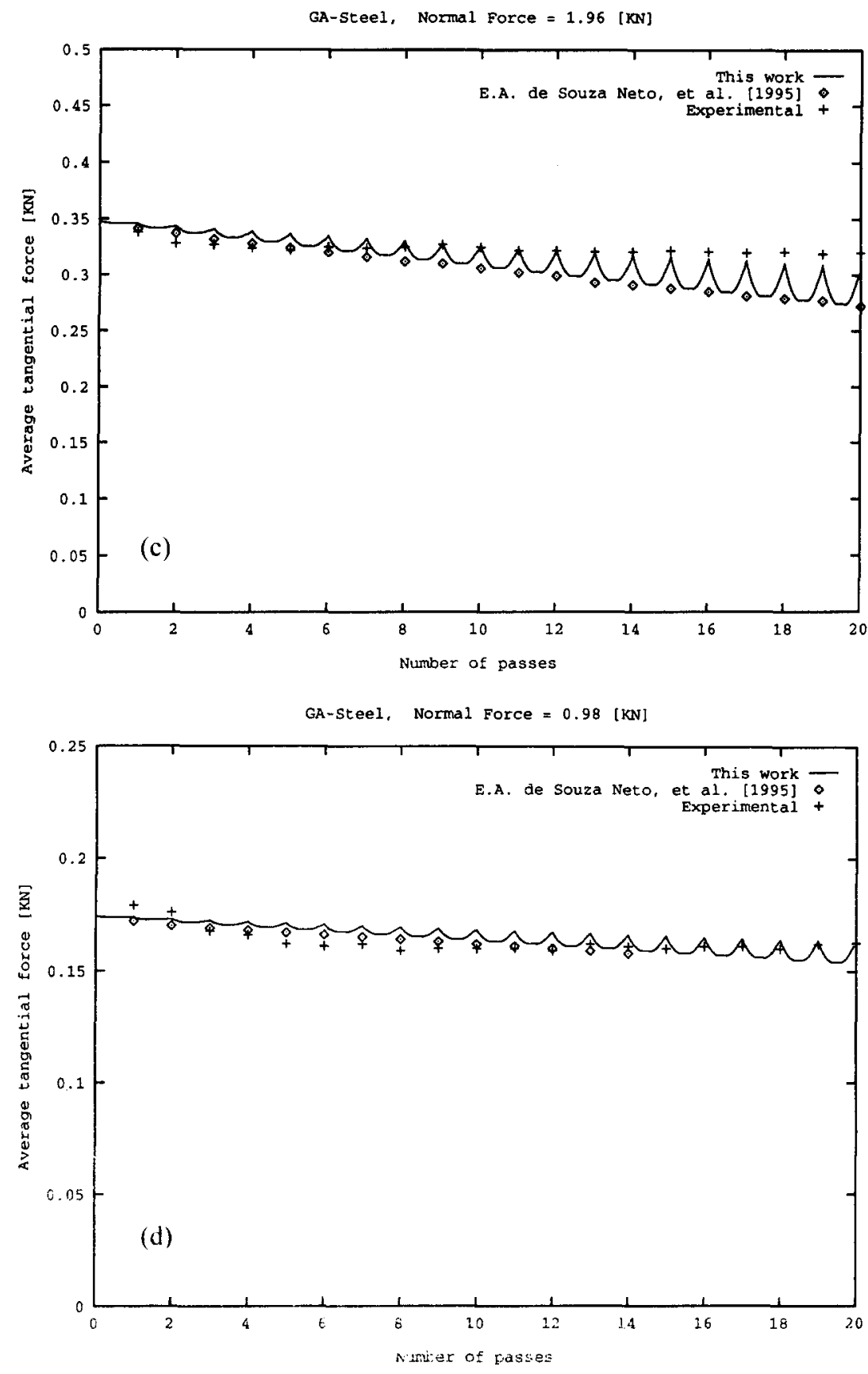

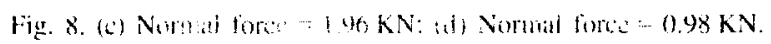


For simplicity, only $30 \mathrm{~mm}$ of the sheet length has been considered in the numerical simulation and a plane strain state has been assumed. The sliding cycle has been repeated 20 times for different compressive constant normal forces of $3.92,2.94,1.96$ and $0.98 \mathrm{KN}$ applied to the tip of the tool.

A mesh of 111 four noded quadrilateral elements has been used for the discretization of the tool. The sheet has been discretized by two layers of 60 continuum elements and the nodes of its left edge have been considered as constrained. A mixed Q1/P0 finite element formulation at finite strain has been used. The table has been considered as rigid.

At the beginning of a sliding cycle, the tip lies at $2.5 \mathrm{~mm}$ from the left edge of the sheet. Starting from this
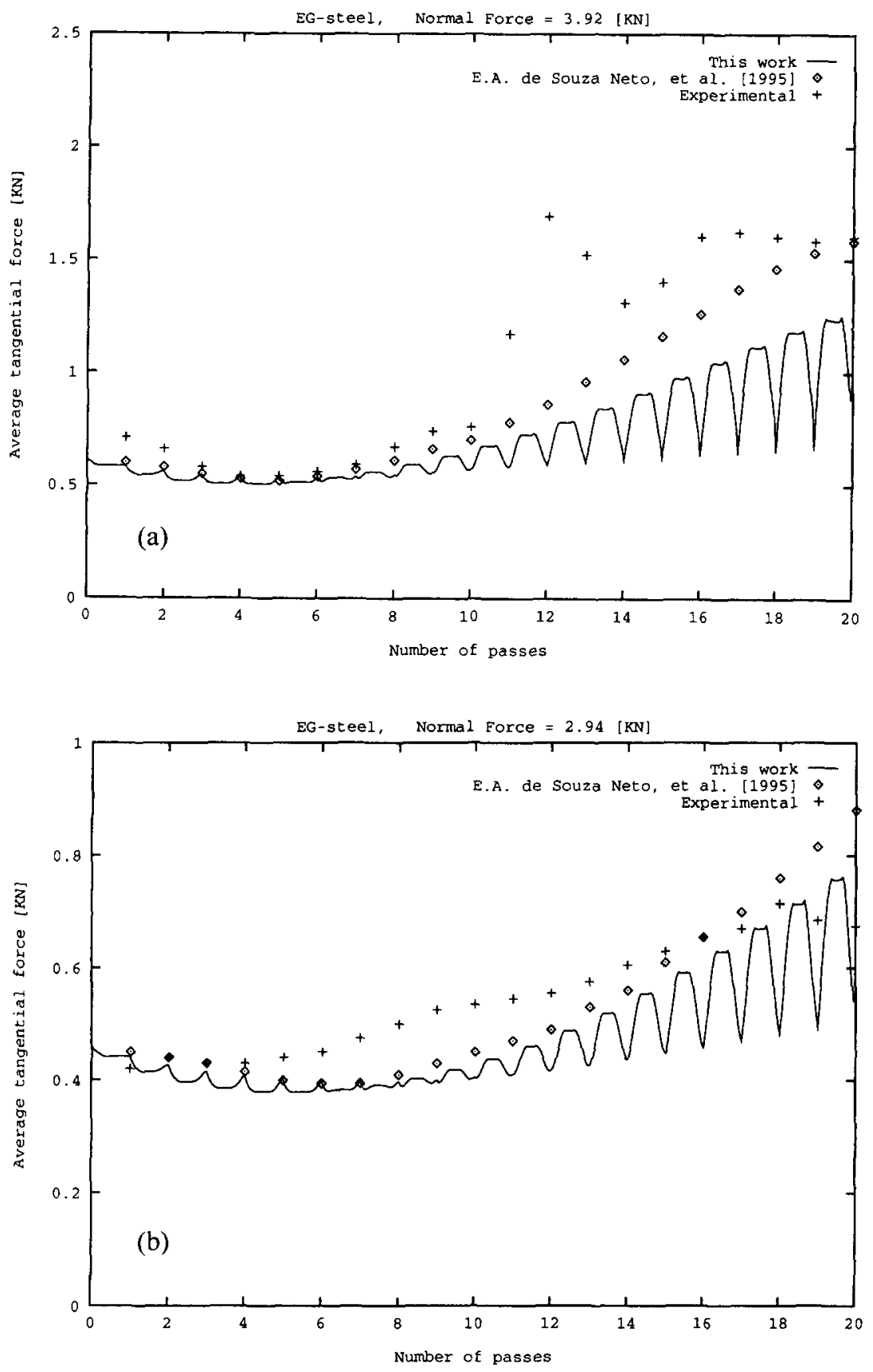

Fig. 9. Flat Sheet Sliding tests. Tangential force versus number of passes during the sliding tests using a EG steel sheet at different constant normal forces: (a) Normal force $=3.92 \mathrm{KN}$; (b) Normal force $=2.94 \mathrm{KN}$. 
initial configuration and after the normal force has been applied, a relative sliding of $20 \mathrm{~mm}$ between the table and the tip is incrementally imposed. This ensures an approximately $10 \mathrm{~mm}$ long evenly worn region on the sheet surface (between $12.5 \mathrm{~mm}$ and $22.5 \mathrm{~mm}$ from the left edge). Then the normal force is released, the tip is lifted up and returned to its initial position, thereby closing a cycle. Note that a steady state frictional force will occur when the entire surface of the tip contacts the evenly worn region of the sheet. The finite element mesh as well as the description of a sliding cycle is shown in Figure 7.

Frictional contact constraints were regularized by means of penalty method and the normal and tangential penalty parameters were taken as $\varepsilon_{N}=5 \times 10^{11} \mathrm{~N} / \mathrm{m}^{3}$ and $\varepsilon_{T}=1 \times 10^{10} \mathrm{~N} / \mathrm{m}^{3}$, respectively. A typical loading
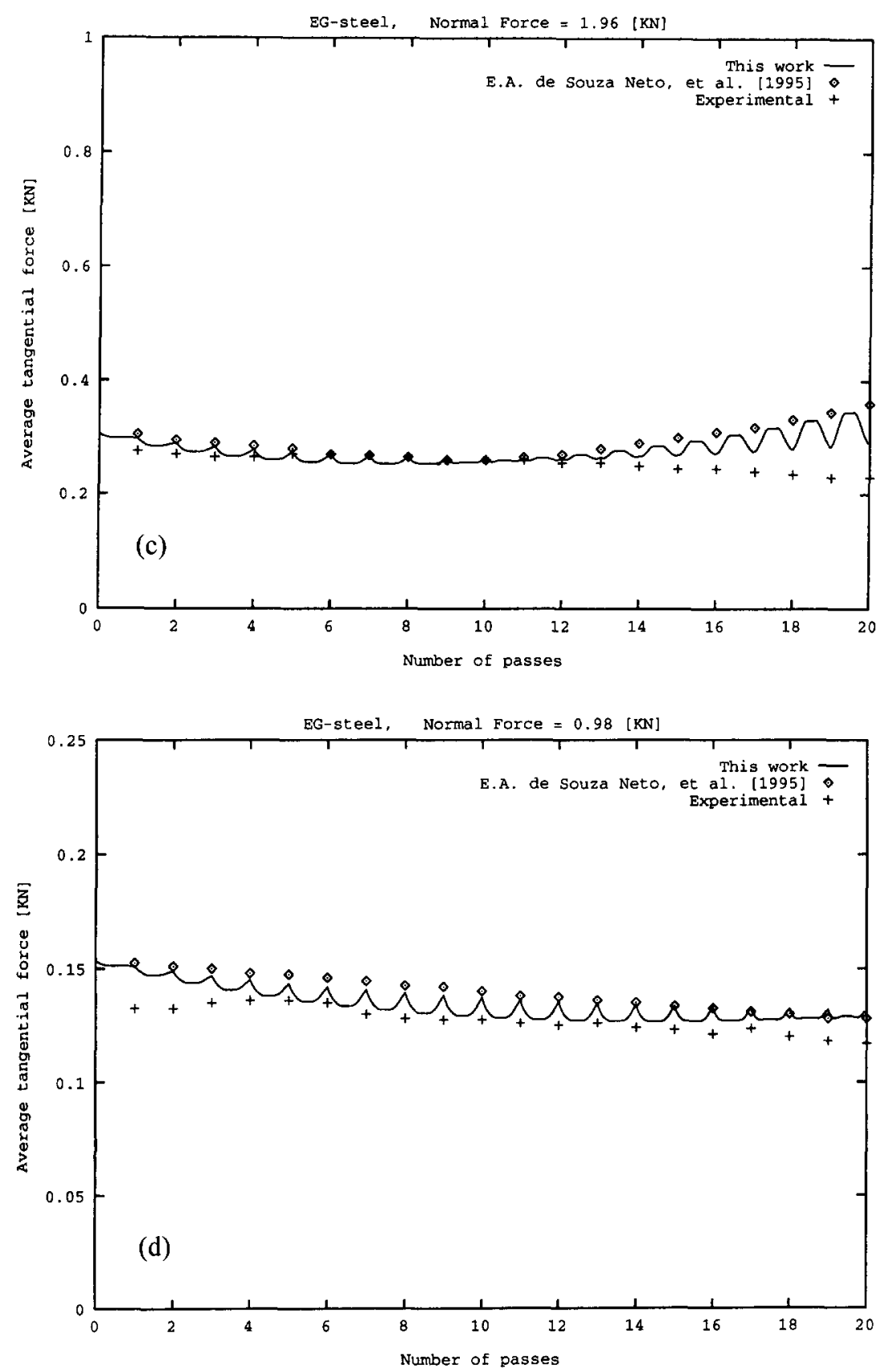

Fig. 9. (c) Normal force $=1.96 \mathrm{KN}$; (d) Normal force $=0.98 \mathrm{KN}$. 
cycle was achieved in 30 time steps: 5 steps to apply the normal force, 20 steps for sliding and 5 steps to remove the normal force.

The Newton-Raphson method, combined with a line search optimization procedure, was used to solve the nonlinear system of equations arising from the spatial and temporal discretization of the weak form of the momentum balance equation. Convergence of the incremental iterative solution procedure was monitored by requiring a tolerance of $10^{-18}$ in the energy norm.

The analysis was performed on a Silicon Graphics Power Challenge L Workstation and a typical case, GA steel sheet using a normal force of $0.98 \mathrm{KN}$, was accomplished in $23 \mathrm{~min}$ CPU time. Table 4 shows the Euclidean norm of the residual at four typical time steps, using a GA steel and a normal force of $0.98 \mathrm{KN}$. A typical intermediate step at different passes has been selected.

Figs. 8 and 9 show the tangential forces obtained in the numerical analysis of the sliding tests, using GA steel and EG steel sheets, respectively, at different constant normal forces. In order to compare the results obtained in this work with the (average) experimental and numerical results given by de Souza Neto et al. [12], it is important to observe that one must consider only an average value within the central part of the sheet for each pass, in the evenly worn region, disregarding the values at the beginning and at the end of each pass, where the distribution of the friction coefficient is not uniform. A detail of the wear profile in the sheet is depicted in Fig. 10 , for the GA steel and for a normal force of $0.98 \mathrm{KN}$. The figure clearly shows an evenly worn region in the central part of the sheet, between 12.5 and $22.5 \mathrm{~mm}$ from the left edge, while the wear at the edges is not uniform.

In Figs. 8 and 9, it is clearly evident the different wear evolution experimented by the GA and EG steel sheets. For the GA steel, due to the softening of the friction coefficient law, the tangential force presents a local minimum within a pass at the central part of the sheet, in the evenly worn region. In contrast, for the EG steel, particularly for high normal pressures, the tangential force at the central part of the sheet moves from a local minimum towards a local maximum within a pass, according to the frictional softening/hardening behavior. Remarkably, a significant hardening is observed for the EG steel at high normal pressures, while a slight softening appears at low normal pressures. These results clearly show that a classical frictional Coulomb law, using a constant friction coefficient, would not be able to capture this behavior, leading to useless inaccurate predictions.

The tangential forces predicted by the numerical analyses, for both GA and EG steel sheets and for all levels of constant normal force, agree well with the experimental and numerical (average) results given by de Souza Neto et al. [12].
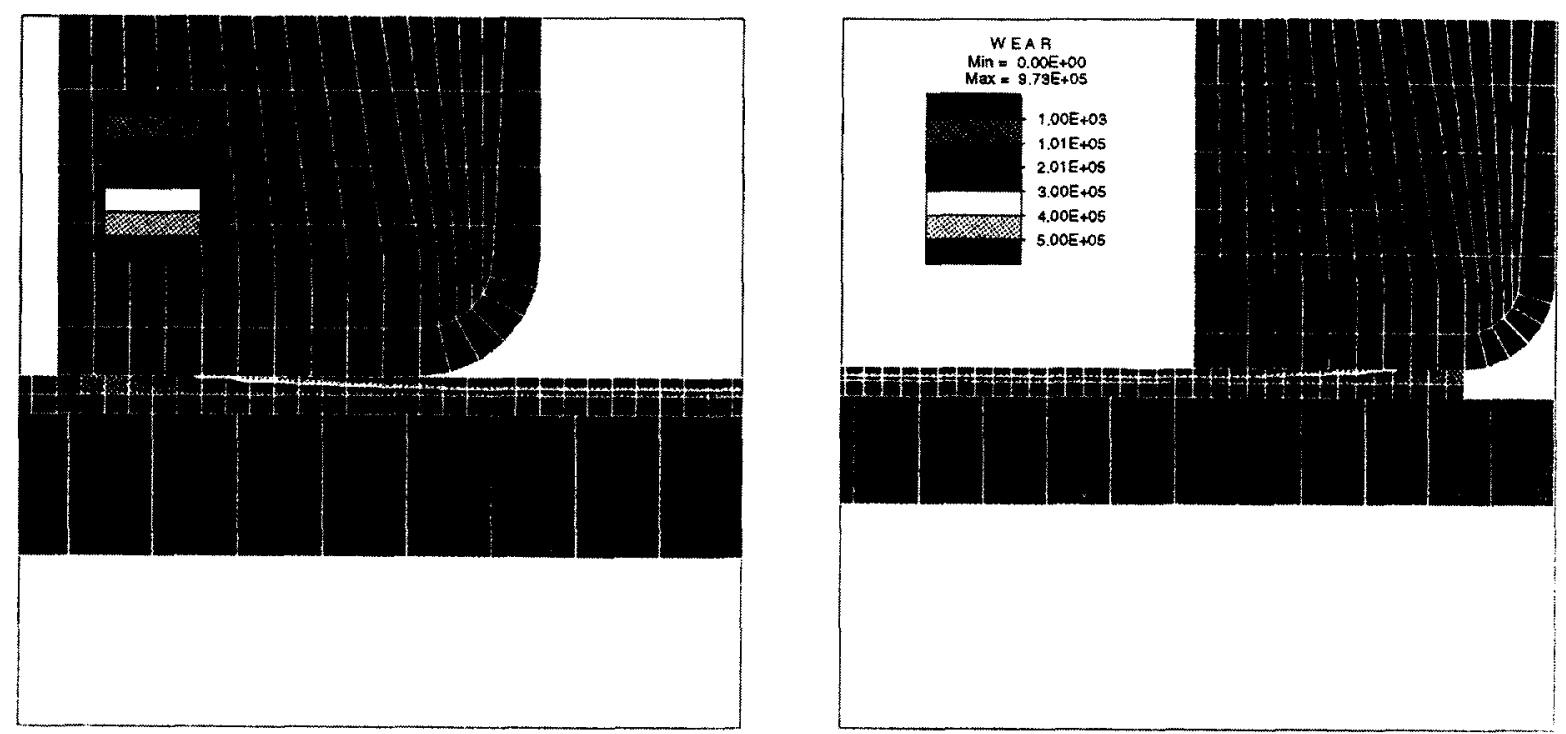

Fig. 10. Flat Sheet Sliding tests. Wear profiles at the GA steel shect for a normal force of $0.98 \mathrm{KN}$. 


\section{Concluding remarks}

Wear related phenomena have an important impact on the economy of industrial metal forming processes. Wear is the dominant die failure mechanism for both bulk and sheet forming operations. The inclusion of wear phenomena model into available decision support systems used in industrial design and optimization practice, would improve die design and service life considerably, leading to an important reduction of manufacturing costs.

Adhesive and abrasive wear have been identified as the main wear mechanisms. Archard's wear law provides an estimate of both wear mechanisms arising in metal forming operations. The law uses the assumption that the wear volume is: proportional to the sliding length, proportional to the normal pressure, inversely proportional to the yield strength (hardness) and proportional to a constant, mainly dependent on material combination, lubricant and temperature for adhesive wear, and surface topology, hard particles between surfaces and lubricant for abrasive wear.

Clearly, wear affects the frictional conditions between contact surfaces. A frictional wear contact model has been proposed, taking the frictional coefficient as a function of a wear related internal variable, to be chosen as the frictional dissipation or the slip amount. This frictional wear model has been incorporated to a continuumbased multi-body frictional contact formulation at finite strains.

Within the context of the displacement-driven formulation of frictional contact problems, exploiting the computational framework developed for plasticity, two frictional return mapping algorithms have been considered: the BE and the implicit PMP rules. An exact linearization of the algorithms allows to derive the consistent frictional contact tangent operator.

Numerical simulations show the suitability of the proposed model to predict wear phenomena. A good agreement has been observed between the numerical results and the experimental ones obtained for the sliding tests. These results allows to suggest that the adoption of the frictional dissipation as the internal variable associated to the frictional behavior has captured the essential features of the wear phenomena.

\section{Acknowledgement}

Support for this work was provided by the European Commission through the BRITE-EURAM Project no. BE-5248-92, under contract no. BRE2-CT92-0180. This support is gratefully acknowledged.

\section{References}

[1] J.T. Oden and J.A.C. Martins, Models and computational methods for dynamic friction phenomena, Comput. Methods Appl. Mech. Engrg. 52 (1985) 527-634.

[2] T.A. Laursen and J.C. Simo, On the formulation and numerical treatment of finite deformation frictional contact problems, in: P. Wriggers and W. Wagner, eds., Nonlinear Computational Mechanics-State of the Art (Springer-Verlag, Berlin, 1991) 716-736.

[3] T.A. Laursen and J.C. Simo, Formulation and regularization of frictional contact problems for Lagrangian finite element computations, in: D.R.J. Owen, E. Onate and E. Hinton, eds., Proc. Third Int. Conf. on Computational Plasticity: Fundamentals and Applications, COMPLAS III (Pineridge Press, Swansea, 1992) 395-407.

[4] T.A. Laursen and J.C. Simo, A continuum-based finite element formulation for the implicit solution of multi-body, large deformation frictional contact problems, Int. J. Numer. Methods Engrg. 36 (1993) 3451-3485.

[5] T.A. Lauren and J.C. Simo. Algorithmic symmetrization of Coulomb frictional problems using augmented Lagrangians, Comput. Methods Appl. Mech. Engrg. 108 (1993) 133-146.

|6| J.C. Simo and T.A. Laursen. An augmented Lagrangian treatment of contact problems involving friction, Comput. Struct. 42 (1992) $97-116$.

(7) J.C. Simo. Numerical analysis aspects of plasticity, in: P.G. Ciarlet and J.J. Lions, eds. Handbook of Numerical Anitlysis, Vol. IV (North-Holland, 1994).

[8] C. Agelet de Saracibar, A new frictional time integration algorithm for multi-body large slip frictional contact problems. Comput. Methods Appl. Mech. Engrg. 142 (1997) 303-334.

[9] S. Lassen. Formulation of weat models for forming dies. Inernal Report, IPU, Technical University of Denmark. 1993.

[10] S. Lassen and N. Bay. Evaluation of tribology tests for sheet metal forming, Internal Report. IPU. Technical University of Denmark, 1993. 
[11] D.R.J. Owen, D. Perić, A.J.L. Crook, E.A. de Souza Neto, J. Yu and M. Dutko, Advanced computational strategies for 3D large scale metal forming simulations, Proc. Fifth Int. Conf. on Numerical Methods in Industrial Forming Processes (A.A. Balkema, 1995),

[12] E.A. de Souza Neto, K. Hashimoto, D. Perić and D.R.J. Owen, A phenomenological model for frictional contact of coated steel sheets, Int. J. Materials Processing Technology 50 (1995) $1-4$.

[13] N. Stromberg, L. Johansson and A. Klarbring, Derivation and analysis of a generalized standard model for contact, friction and wear, Int. J. Solids Struct. 33 (1996) 1817-1836.

[14] N. Stromberg, Thermomechanical modelling of tribological systems, Ph.D. Thesis, Report no. 497, Linkoping Studies in Science and Technology, 1997.

[15] J.F. Archard, Contact and rubbing of flat surfaces, J. Appl. Phys. 24 (1953) 981-988.

[16] O.C. Zienkiewicz and R.L. Taylor, The Finite Element Method, 4th edition, Vol. 2: Solid and Fluid Mechanics, Dynamics and Non-linearity (McGraw-Hill, London, 1991).

[17] N. Kikuchi and J.T. Oden, Contact Problems in Elasticity: A Study of Variational Inequalities and Finite Element Methods (SIAM, Philadelphia, 1988).

[18] G. Duvaut and J.L. Lions, Les Inequations en Mecanique et en Physique (Dunod, Paris, 1972).

[19] J.C. Simo, Algorithms for static and dynamic multiplicative plasticity that preserve the classical return mapping schemes of the infinitesimal theory, Comput. Methods Appl. Mech. Engrg. 99 (1992) 61-112.

[20] A. Thomas, Investigations on die materials, Ph.D. Thesis, Drop Forging Research Assocoatopm. Sheffield, UK, 1970.

[21] K. Miyauchi et al., Galling behaviour and its tests in press forming of autobody parts, IDDRG 10th Congress (1978) $287-296$.

[22] T. Abildgaard, Continuummekanisk og metallurgisk undersogelse af abrasive procesmekanismer, Report MM-85.32, Technical University of Denmark, 1985. 